\title{
Spin Readout of a CMOS Quantum Dot by Gate Reflectometry and Spin-Dependent Tunneling
}

\author{
Virginia N. Ciriano-Tejel $\odot,{ }^{1,2,}{ }^{*}$ Michael A. Fogarty $\odot,{ }^{1,2}$ Simon Schaal® ${ }^{1,2}$ Louis Hutin $\odot,{ }^{3}$ \\ Benoit Bertrand๑, ${ }^{3}$ Lisa Ibberson $\odot,{ }^{4}$ M. Fernando Gonzalez-Zalba $\odot,{ }^{4},+$ Jing Li $\odot,{ }^{5}$ \\ Yann-Michel Niquet $\odot,{ }^{5}$ Maud Vinet, ${ }^{3}$ and John J.L. Morton $\oplus^{1,2,6, \dagger}$ \\ ${ }^{1}$ London Centre for Nanotechnology, University College London, London WC1H 0AH, United Kingdom \\ ${ }^{2}$ Quantum Motion Technologies, Windsor House, Cornwall Road, Harrogate HG1 2PW, United Kingdom \\ ${ }^{3}$ CEA, LETI, Minatec Campus, F-38054 Grenoble, France \\ ${ }^{4}$ Hitachi Cambridge Laboratory, J.J. Thomson Avenue, Cambridge CB3 OHE, United Kingdom \\ ${ }^{5}$ Université Grenoble Alpes, CEA, IRIG-MEM-L_Sim, F-38000 Grenoble, France \\ ${ }^{6}$ Department of Electronic and Electrical Engineering, UCL, London WC1E 7JE, United Kingdom
}

(Received 16 May 2020; revised 12 October 2020; accepted 24 February 2021; published 31 March 2021)

Silicon spin qubits are promising candidates for realizing large-scale quantum processors, benefitting from a magnetically quiet host material and the prospects of leveraging the mature silicon device fabrication industry. We report the measurement of an electron spin in a singly occupied gate-defined quantum dot, fabricated using CMOS-compatible processes at the 300-mm wafer scale. For readout, we employ spin-dependent tunneling combined with a low-footprint single-lead quantum-dot charge sensor, measured using rf gate reflectometry. We demonstrate spin readout in two devices using this technique, obtaining valley splittings in the range $0.5-0.7 \mathrm{meV}$ using excited-state spectroscopy, and measure a maximum electron-spin relaxation time $\left(T_{1}\right)$ of $9 \pm 3 \mathrm{~s}$ at $1 \mathrm{~T}$. These long lifetimes indicate the silicon-nanowire geometry and fabrication processes employed here show a great deal of promise for qubit devices, while the spin-readout method demonstrated here is well suited to a variety of scalable architectures.

DOI: 10.1103/PRXQuantum.2.010353

\section{INTRODUCTION}

Spin qubits in silicon have been shown to fulfil most of the requirements to realize a quantum computer [1], including high-fidelity qubit manipulation [2], single-shot readout [3-5], and long coherence times [6,7]. Remaining challenges to realize a silicon quantum processor include building on recent demonstrations of two-qubit gates [8-11] to reach the fault-tolerant threshold, as well as showing how scalable control and measurement of silicon qubits can be achieved in a way that is compatible with their high intrinsic density. While hole spin qubits have been demonstrated using CMOS-compatible manufacturing processes based on nanowire field effect transistors

\footnotetext{
*virginia.ciriano.17@ucl.ac.uk

†jjl.morton@ucl.ac.uk

$\$$ Present address: Quantum Motion Technologies, Windsor House, Cornwall Road, Harrogate, HG1 2PW, United Kingdom.

Published by the American Physical Society under the terms of the Creative Commons Attribution 4.0 International license. Further distribution of this work must maintain attribution to the author(s) and the published article's title, journal citation, and DOI.
}

(NW FETs) [12], open questions remain as to how the nanowire and its fabrication in industry standard cleanrooms impact electron-spin properties such as relaxation and coherence times.

Spin-qubit readout in silicon requires a spin-to-charge conversion step followed by charge detection. Various forms of spin-to-charge conversion exist such as Pauli spin blockade (PSB) [13] or spin-dependent tunneling to a reservoir [14]. PSB can be detected dispersively [15-17], but typically charge sensors close to the qubit have been used in combination with both spin-dependent processes [6,7,18-21]. Standard three-terminal charge sensors such as the quantum point contact (QPC) or single-electron transistor (SET) have achieved spin-readout fidelities as high as $99.9 \%$ in $6 \mu \mathrm{s}[22,23]$ in dc mode and $99 \%$ in $1.6 \mu \mathrm{s}$ in $\mathrm{rf}$ mode [4]. However, these sensors require two charge reservoirs near the qubit, complicating the use of this method at scale in dense qubit arrays.

As a more scalable alternative, charge sensors consisting of just two terminals in which a charge island is connected to a single reservoir, i.e., a single-electron box (SEB), have gained considerable traction [3,24-26]. In this method, the complex impedance of a quantum dot, which may contain both dissipative and dispersive contributions [27,28], is 


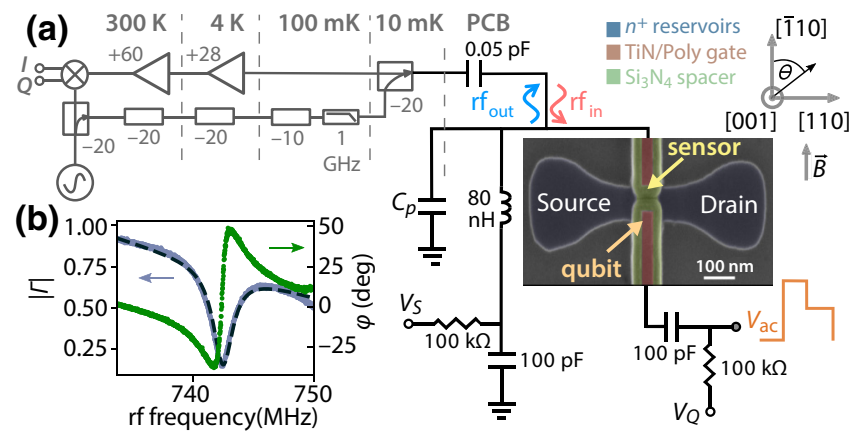

FIG. 1. Device and measurement setup. (a) False-color transmission electron micrograph of a silicon nanowire with a pair of split gates. Quantum dots are formed under each gate, referred to as "sensor" and "qubit" dots, and controlled, respectively, by $V_{S}$ and $V_{Q}$. The sensor dot is connected to a lumped-element resonator for dispersive readout. Fast pulses, $V_{\mathrm{ac}}$, are applied to the qubit dot through a bias tee. To lift the spin degeneracy, a magnetic field is applied in the [110] crystallographic direction, perpendicular to the nanowire. The magnetic field orientation can be rotated in the plane of the device, making an angle $\theta$ to [1 110$]$. (b) Magnitude of the reflection coefficient, $|\Gamma|$, showing the resonator frequency at $0 \mathrm{~T}$. Applying a magnetic field reduces the resonant frequency due to changes in the kinetic inductance of the superconducting inductor that forms the resonator [31], see Appendix B for further details.

measured by connecting a lumped-element resonator either via a gate that controls the dot or via the reservoir. Changes in the surrounding charge environment modify the bias point of the SEB, which in turn produce a rf response conditional to the charge state of the sensed element. A spin-polarized SEB has been used to achieve spin-parity readout with a fidelity of $>99 \%$ in $1 \mathrm{~ms}$ [3]. However, a demonstration of SEB-based single-spin readout is still lacking.

In this paper, we demonstrate time-averaged spin readout of a single electron in a quantum dot through spindependent tunneling, detected using an adjacent quantum dot (charge sensor), which is connected to a gate-based reflectometry setup. The quantum dots are formed on opposite corners of a silicon split-gate NW FET, fabricated using CMOS-compatible processes [Fig. 1(a)]. We perform excited-state spectroscopy of the quantum dot and measure spin relaxation times $\left(T_{1}\right)$ as a function of magnetic field magnitude and orientation. We measure $T_{1}$ up to $9 \pm 3 \mathrm{~s}$ - to our knowledge the longest measured so far for silicon quantum dots. This suggests that the CMOS processes and nanowire geometry do not pose limitations on spin relaxation and hold considerable promise for high-quality qubits compatible with scalable manufacture.

\section{SETUP}

Below, we present spin readout in two NW FET devices, an example of which is shown in Fig. 1(a). Device A has a gate length $L_{g}=50 \mathrm{~nm}$ and nanowire width $W=80$ $\mathrm{nm}$, and device B has $L_{g}=40 \mathrm{~nm}$ and $W=70 \mathrm{~nm}$. Two gates wrap onto the nanowire, in a face-to-face arrangement, with a separation between the gates, $S_{v}$, of $50 \mathrm{~nm}$ for device A and $40 \mathrm{~nm}$ for device B. Each gate can be tuned using a dc voltage to electrically induce quantum dots in the opposite corners of the silicon nanowire [29], while ac signals applied to the gates are used for control and $\mathrm{rf}$ reflectometry readout. The two quantum dots are tunnel coupled (in a parallel configuration) to self-aligned, heavily implanted, $n$-type source and drain electron reservoirs, and capacitively coupled to each other. The device is notionally symmetric; however, we nominate one of the dots the "sensor dot" by connecting its gate to an $L C$ resonator for gate-based reflectometry [30]. Further details of the devices, including fabrication methods, are presented in Appendix A.

By monitoring the phase of the reflected rf signal, while the sensor and qubit potentials $V_{S}$ and $V_{Q}$ are swept, it is possible to map out charge transitions for the two quantum dots [see Fig. 2(d) for detail and Fig. 8 for a full stability diagram]. Because the reflectometry signal is a function of the tunneling rate of the sensor dot to the reservoir, and this rate depends on the sensor-dot occupancy, $n_{s}$, it is not straightforward to assign an electron occupation for this dot [31]. Nevertheless, $n_{s}$ is not central to the charge sensing we employ here. The number of electrons in the qubit dot, $n_{q}$, can be measured using the interdot capacitive coupling with the sensor: each change in $n_{q}$ shifts the sensor-dot electrochemical potential [see Fig. 2(d)] allowing us to ensure complete depletion in the qubit dot by reducing $V_{Q}$ until no further shifts are observed by the sensor (see Fig. 8).

\section{SPIN READOUT}

Once the qubit dot is depleted to its last electron, the spin degeneracy is lifted by applying a magnetic field in the plane of the device and perpendicular to the nanowire, in the [110] crystallographic direction. The spin-readout procedure follows a three-level pulse applied to the gate forming the qubit dot, cycling between three states: load-emptyread marked as L, E, and R, respectively, in Fig. 2(a). The potential of the "read" state sits between "load" and "empty," at the $0 \leftrightarrow 1$ charge transition for $n_{q}$, such that the Fermi energy of the reservoir lies between the Zeemansplit spin $|\uparrow\rangle$ and $|\downarrow\rangle$ states [14]. At this point, a spin $|\downarrow\rangle$ electron remains in the qubit dot, while a spin $|\uparrow\rangle$ electron tunnels out to the reservoir, to be subsequently replaced by a spin $|\downarrow\rangle$ electron tunneling on the qubit dot. This spin-dependent tunneling is detected using the sensor dot when tuned to a point in the stability diagram where the reflectometry signal depends on the qubit-dot electron occupation. Usable "read" points in the stability diagram are ones where the $n_{q}=0 \leftrightarrow 1$ charge transition 


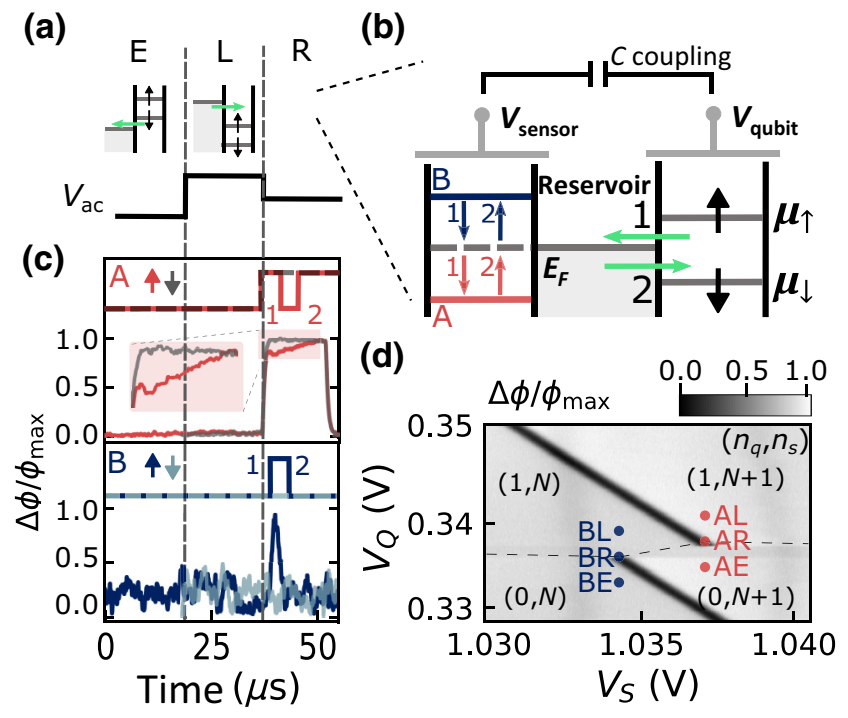

FIG. 2. Spin readout. (a) Three-level pulse sequence applied to the qubit-dot gate: first emptying the dot (E), then loading an electron with a random spin orientation into the dot (L), and finally reading the spin state $(\mathrm{R})$. (b) For spin readout, the qubitdot potential is tuned so its spin-up and spin-down states straddle the reservoir Fermi energy. A spin-down electron remains in the qubit dot, whereas a spin-up electron tunnels out (1) followed by a spin-down electron entering the dot (2). Due to capacitive interdot coupling, changes in the qubit-dot charge state cause the sensor-dot electrochemical potential to shift into or out of alignment with the reservoir, leading to the appearance or suppression of a phase response signal $\Delta \phi$ in reflectometry. (c) Single-shot schematics and time-averaged measured phase response (1024 averages) for device A (red), and B (blue), where the spin-up signature is, respectively, a dip or a peak in the phase response. (d) Charge stability diagram of the double quantum dot near the $\left(n_{q}, n_{s}\right)=(1, N) \leftrightarrow(0, N+1)$ charge transition for device $\mathrm{B}$ (device A measurements use a nominally identical charge transition). Only the sensor-dot lead-to-dot transition is visible in reflectometry.

intersects with the $n_{s}=N \leftrightarrow N+1$ transition that yields a reflectometry signal. Two such points can be identified in Fig. 2(d) labeled AR and BR. At "BR," a reflectometry signal (arising from the $n_{s}=N \leftrightarrow N+1$ transition) is visible only when the qubit dot is empty $\left(n_{q}=0\right)$. In this case, the signature of a spin $|\uparrow\rangle$ electron on the qubit dot is the brief emergence of a reflectometry signal at the read point, as the electron tunnels out of the dot (and a new spin $|\downarrow\rangle$ tunnels in). Conversely, at "AR," a reflectometry signal is visible only when the qubit dot is occupied $\left(n_{q}=1\right)$, in which case the signature of spin $|\uparrow\rangle$ is a transient reduction in the signal. Experiments on device B used point BR for readout, while those on device $A$ used a point equivalent to AR in the device A stability diagram. Figure 2(c) shows the ideal and measured spin-readout traces averaged over 1024 "ELR" cycles at both "AR" and "BR." Further tests of spin readout are shown in Appendix D.
Detecting the spin-dependent transient signals requires that the tunneling rate $\Gamma_{0}$ between the qubit dot and reservoir falls within the resonator bandwidth. The resonator $Q$ factor in our experiments is magnetic field dependent leading to a detection bandwidth in the range $1.5-4.0 \mathrm{MHz}$ (See Appendix B). Dot-to-reservoir tunneling rates in these devices can be tuned by applying a voltage to a global metal top gate [not shown in Fig. 1(a)] or to the substrate [26,32]. We apply 0 and $-10 \mathrm{~V}$ to the metal top gate for devices $\mathrm{A}$ and $\mathrm{B}$, respectively, with the substrate at $0 \mathrm{~V}$, to achieve suitably low tunneling rates: $\Gamma_{0, g_{A}}=0.62(1)$ $\mathrm{MHz}$ for device A and $\Gamma_{0, g_{B}}=0.97(1) \mathrm{MHz}$ for device B. The larger tunneling rates in device B makes "BR" a more optimal set point for the readout visibility. The spin-readout signal is further optimized by fine tuning the sensor and qubit-gate voltages $V_{S}$ and $V_{Q}$. Through simulations of the signal dependence on these voltages, arising from the energy-dependent tunneling rates [see Eq. (1) and Appendix C], we obtain the tunneling rates quoted above, as well as an estimated $g$ factor of $g=1.92(11)$, and the qubit-dot effective temperature of 230(9) $\mathrm{mK}$ (for device B). This temperature limits the minimum Zeeman splitting at which spin readout is feasible and its elevation compared to the device temperature $(10 \mathrm{mK})$ is attributed to the influence of the rf readout signal applied to the sensor dot acting on the qubit dot. The rf power used for readout $(-83 \mathrm{dBm})$ can be decreased, albeit with a reduction in the spin-up visibility (see Appendix F), however, amplifiers operating at the quantum limit of introduced noise can be used to achieve higher sensitivity in rf reflectometry while using lower drive powers [33].

\section{SPIN RELAXATION}

We next consider the effect of spin relaxation by varying the duration of the "load" period in the three-level pulse sequence. The spin of the loaded electron relaxes from its initial randomized state into the spin $|\downarrow\rangle$ ground state with a time constant $T_{1}$. We observe exponential decays in the spin $|\uparrow\rangle$ fraction (see Appendix $\mathrm{H}$ ), which we fit to obtain relaxation rates $T_{1}^{-1}$, plotted in Fig. 3 as a function of magnetic field strength and orientation. In both devices, we observe an increase in $T_{1}$ as the magnetic field is decreased up to a maximum of $T_{1}=0.28(3) \mathrm{s}$ (device A) and $T_{1}=9(3) \mathrm{s}($ device B) at $B=1 \mathrm{~T}$.

The magnetic field dependence of $T_{1}$ varies according to the relaxation mechanism and the direction of the field with respect to crystal axes. For the measurements presented in Fig. 3(a) the magnetic field is parallel to [110]. Spin relaxation may arise from magnetic noise at the spin Zeeman frequency or, more commonly and given some spin-orbit coupling (SOC) that mixes the spin degree of freedom with orbital or valley states, from phonon-induced electric field noise or Johnson noise. At this field orientation, and far from any anticrossing with higher-lying excited 

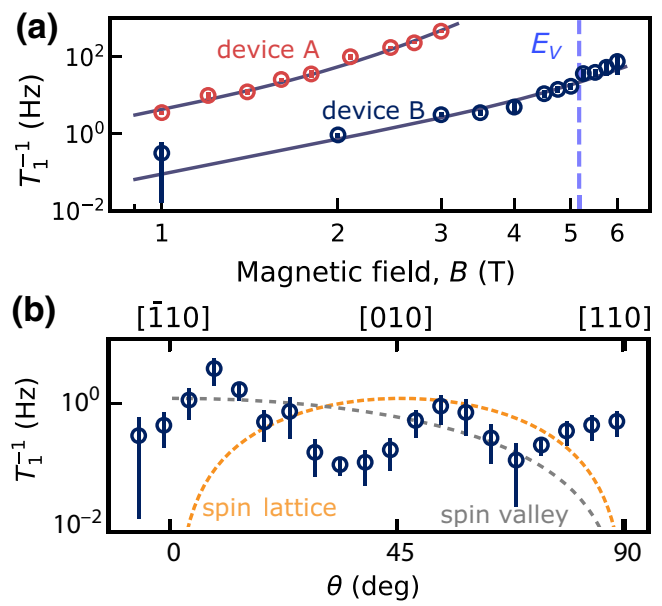

FIG. 3. Spin-relaxation rates and their magnetic field dependence. (a) Relaxation rate measured with the magnetic field applied perpendicular to the nanowire, in the plane of the device, in the [110] crystallographic direction. Curves are fits to a general model described in the text, and $E_{V}$ marks the field at which the Zeeman splitting matches the measured valley splitting in device B. (b) Dependence of $T_{1}^{-1}$ on magnetic field orientation at $1 \mathrm{~T}$ for device $\mathrm{B}$, where $\theta$ is the angle between the magnetic field and the $[\overline{1} 10]$ crystallographic direction for device $B$ in the nanowire plane. The angular dependence expected from spin-valley mixing in an ideal corner dot (dashed gray curve) is insufficient to explain the observed trend. Spin-lattice relaxation mechanisms can, however, give rise to higher-order angular modulations [34] in quantum dots with high symmetry (see, for example, orange dashed curve and Appendix J).

states [35], the primary contributions from phonons to the relaxation rate $T_{1}^{-1}$ are proportional to $B^{7}[36,37]$, while those from Johnson-Nyquist noise are proportional to $B^{3}$ [36]. We therefore fit the data in Fig. 3(a) to a combination of such processes: $T_{1}^{-1}=c_{\mathrm{ph}} B^{7}+c_{J} B^{3}$ (see Appendix J).

We study the angular dependence of the spin-relaxation rate in device $\mathrm{B}$, rotating a $B=1 \mathrm{~T}$ field in the plane of the device. A minimum in the relaxation rate is expected as the magnetic field is parallel to the direction of the nanowire, aligned along the [110] crystallographic direction since there is no spin-valley mixing (a typically dominant spin-orbit mixing mechanism) when the magnetic field is perpendicular to a mirror symmetry plane of the device $[37,38]$. Although we see a downward trend towards [110], we find that the usual models for spinorbit driven relaxation $[34,37,39,40]$ [see dashed lines in Fig. 3(b)] are not able to account for all features in the angular dependence.

In general though, spin-lattice relaxation can produce higher-order harmonics in the dependence on magnetic field orientation, especially in quantum dots with high inplane symmetry (see Appendix J). Such a high symmetry in device B would also suggest a weak spin-valley mixing with implications on the relaxation behavior when the
Zeeman splitting becomes comparable to the excited-state valley splitting.

\section{EXCITED-STATE SPECTROSCOPY}

To gain further insights into the spin-relaxation mechanism for this device, we move on to study the excited states of the quantum dot by sweeping the voltage of the "load" stage, $V_{Q, L}$. The rate at which an electron loads from the reservoir into some dot state $|i\rangle$ depends on the difference in electrochemical potential, $\Delta E_{i}$, between $|i\rangle$ and the reservoir Fermi energy. Here, we consider four dot states, $i \in\left\{g_{\downarrow}, g_{\uparrow}, e_{\downarrow}, e_{\uparrow}\right\}$, where $g$ and $e$ are, respectively, the ground and excited $z$-valley states, each with spin-up and spin-down states. Assuming elastic tunneling and a constant reservoir density of states, the loading rate follows a Fermi-Dirac distribution centered at $\Delta E_{i}=0$, when dot and lead potentials are aligned $[41,42]$ :

$$
\Gamma_{i}^{\text {load }}=\frac{\Gamma_{0, i}}{1+e^{\Delta E_{i} / k_{B} T}},
$$

where $\Gamma_{0, i}$ is the natural tunnel rate for each state $|i\rangle, k_{B}$ is the Boltzmann constant, and $T$ the effective temperature. We assume here that the natural tunnel rates are spin independent (i.e., for the ground states $g_{\downarrow}$ and $g_{\uparrow}$ they are equal to $\Gamma_{0, g}$, and similarly for the excited-state naturaltunnel rate $\Gamma_{0, e}$ ), as well as independent of $V_{Q, L}$ over the small (approximately $1 \mathrm{mV}$ ) range of voltages studied here. The energy separation $\Delta E_{i}$ can be tuned with $V_{Q, L}$ as $\Delta E_{i}=|e| \alpha_{Q Q}\left(V_{i}-V_{Q, L}\right)$, where $V_{i}$ is the voltage at which the dot state $|i\rangle$ and reservoir potential align and $\alpha_{Q Q}$ is the gate lever arm of the "qubit gate" to the qubit dot. From Eq. (1), tunneling rates tend to zero for load voltages smaller than $V_{i}$, and towards the natural tunneling rate, $\Gamma_{0, i}$, for higher voltages. As a result, varying the "load" voltage $V_{Q, L}$ changes the tunneling rates into the various dot states, and thus the probability of loading a spin up, which we detect using the spin-readout described above.

To perform excited-state spectroscopy on the qubit dot we use a four-level pulse sequence ("empty"-"load""plunge"-"read") applied to the qubit-dot gate [18], where the additional "plunge" stage ensures that an electron is always loaded for any cycle, while the loading voltage is swept between the "empty" and "plunge" levels [see inset in Fig. 4(a)]. We define a spin-up fraction $P_{\uparrow}$ based on the integrated spin-up signal, baseline corrected, and normalized to obtain $P_{\uparrow}=0.5$ in the limit of zero load time (to neglect relaxation) and random loading using only the "plunge" phase. The dependence of $P_{\uparrow}$ on the "load" voltage (converted to energy) is shown in Fig. 4(a), and can be understood by considering the schematics in Fig. 4(b). In the limit (I) of low $V_{Q, L}$, no electron tunnels into the qubit dot during the "load" phase and an electron of random spin is loaded during "plunge." When the Fermi energy, $E_{F}$, of the reservoir lies between the spin-up and spin-down states 

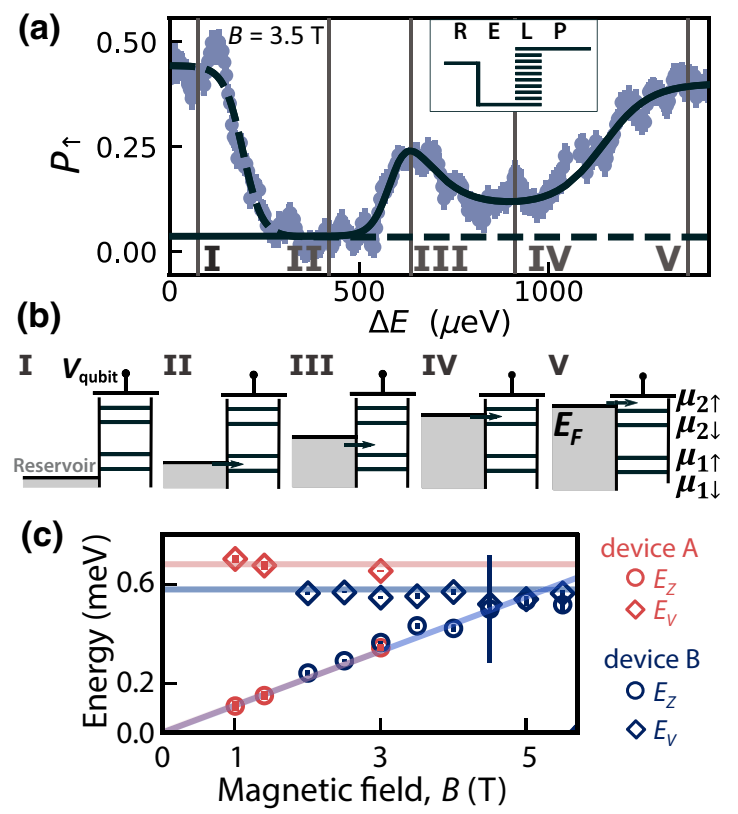

FIG. 4. Excited-state spectroscopy. (a) Measured spin-up fraction for different load levels obtained using energy-selective loading in a four-level pulse scheme as shown in the inset and fit. (b) Illustration of different loading level regimes. (I) When the load level is too low, no electrons are loaded, and an electron with random spin tunnels in during the plunge stage. (II) If the reservoir $E_{F}$ is placed between the spin-up and -down state, only spin-down electrons tunnel in. (III) At higher load levels, a random spin tunnels in during the load stage. (IV) When $E_{F}$ lies between the spin-up and -down levels of the excited state, an electron can occupy any spin state of the ground state and the spin-down excited state. Assuming fast spin-conserving relaxation from the excited to the ground state, most of the electrons are found with spin down. (V) For even higher load levels, the electron tunnels into any possible state. (c) Zeeman splitting $E_{Z}$ and excited-state energy $E_{V}$ obtained by fitting (a) to Eq. (3) at different magnetic fields for devices $\mathrm{A}$ and $\mathrm{B}$.

(II), only spin-down electrons tunnel into the dot, and $P_{\uparrow}$ drops to zero. Assuming the duration of the "load" period in the pulse sequence is long compared to the natural tunneling rates $\Gamma_{0, i}$, the transition between regions I and II is characterized by the spin-down ground-state loading rate, $\Gamma_{\text {load, } g \downarrow}$ :

$$
P_{\uparrow}=\frac{1}{2}\left(1-\frac{\Gamma_{g \downarrow}^{\mathrm{load}}}{\Gamma_{0, g}}\right),
$$

used to generate the dashed curve in Fig. 4(a).

As the "load" voltage is further increased (III), both spin states can be loaded and the measured spin-up fraction increases. Excited states can also be measured in this way provided their decay rates to the ground state are sufficiently high $[36,43]$. Once the spin-down excited state becomes available during the load process (IV), the measured spin-up fraction again reduces, since the excited state rapidly decays in a spin-conserving manner [44]. Finally, in region $(\mathrm{V})$, an electron of either spin orientation can be loaded into the excited state. In regions II-V, the measured spin-up fraction can be modeled by combining all relevant rates [44]:

$$
P_{\uparrow}\left(V_{Q, L}\right)=\frac{\sum_{i=\{g \uparrow, e \uparrow\}} \Gamma_{i}^{\text {load }}\left(V_{Q, L}\right)}{\sum_{i=\{g \uparrow, g \downarrow, e \downarrow, e \uparrow\}} \Gamma_{i}^{\text {load }}\left(V_{Q, L}\right)} .
$$

By fitting the data to Eq. (3) [see solid line in Fig. 4(a)] we can extract several parameters: (i) The Zeeman splitting $E_{Z}$ between the spin-up and spin-down states (fixed to be the same for the ground and excited valley states), related to the width of regions II and IV; (ii) the valley splitting $E_{V}$, related to the separation of regions II and IV; (iii) the ratio between ground- and excited-state natural-tunneling rates, $\Gamma_{0}^{e} / \Gamma_{0}^{g}$, related to the amplitude in region IV; and (iv) the effective temperature $T$, related to the sharpness of transitions between various regions (which can be seen to be different for the ground and excited states, as discussed further in Appendix G).

Extracted values for $E_{Z}$ and $E_{V}$ for both devices are shown in Fig. 4(c) as a function of magnetic field. As expected, $E_{Z}$ shows a linear dependence with field with a $g$ factor of 1.91(10), while $E_{V}$ is field independent and measured to be $0.68(2) \mathrm{meV}$ (device A) and $0.57(3) \mathrm{meV}$ (device B). These values are broadly similar (within a factor of 2) to those measured in similar nanowire devices [3] - furthermore, a large valley splitting is beneficial for spin qubits to remain within the computational basis states and maximize spin-relaxation times [45]. The valley splitting in device $\mathrm{B}$ is shown as an equivalent magnetic field in Fig. 3. When $E_{Z} \sim E_{V}$, there is a finite intervalley spinorbit matrix element leading to spin-valley mixing that should produce a "hot spot" in the relaxation rate. The absence of such a "hot spot" within our measurement resolution $(250 \mathrm{mT})$ sets an upper limit for the "hotspot" linewidth, and, in turn, for the spin-valley mixing strength [46]. Several previous studies have reported wider linewidths, corresponding to stronger spin-valley mixing [35,47,48], however, similar [49], and narrower [45] linewidths have been observed: this places our measurements amongst the lowest in terms of spin-valley mixing strength.

A possible explanation for the weak spin-valley mixing is that the corner dot has greater symmetry than expected, with two orthogonal quasisymmetry planes [38] - this would be consistent with the complex magnetic fieldorientation dependence of $T_{1}$ discussed above. Another possible explanation is phase cancellations between the valley coupling and spin-orbit coupling matrix elements strongly suppressing spin-valley mixing [50,51]. In both cases, this interesting regime warrants investigation of further devices to ascertain the relationship between these conditions and the device geometry, growth conditions, 
and electrostatic environment. The possibility that the excited state probed in this experiment is an orbital rather than a valley excitation, although unlikely, is explored in Appendix I.

In the case of device $A$, we could not confirm the strength of the spin-valley mixing since the excited state lies above our magnet range (6 T). However, a larger spinvalley mixing would explain the shorter relaxation times measured. Given that both devices are measured with the same setup, we suspect that the different relaxation times could arise from the fact that device B is measured with a higher voltage applied to the sensor gate, pulling the qubit-dot wave function outwards from the edge making it more symmetrical. Also consistent with a more symmetric wave-function dot is that device $\mathrm{B}$ is shorter than device A ( $L_{g}=40 \mathrm{~nm}$ against $\left.L_{g}=50 \mathrm{~nm}\right)$. Moreover, we cannot discard that the source of the extra symmetry in device B is due to some fabrication inhomogeneity.

\section{CONCLUSIONS AND OUTLOOK}

We demonstrate time-averaged readout of a single spin confined in a CMOS quantum dot, using a nanowire device fabricated at the $300-\mathrm{mm}$ wafer scale. We introduce a spin-readout method based on spin-dependent tunneling combined with gate-based reflectometry of a neighboring quantum dot to act as a charge sensor, representing a low-footprint approach to spin readout in silicon devices.

Our detector bandwidth and tunnel coupling of the sensor dot to the reservoir would permit spin readout on the timescale of $10 \mu \mathrm{s}$. However, further improvements in the SNR of the gate-based reflectometry are required to achieve high-fidelity single-shot measurements on such short timescales $[4,24,52]$. For a charge transition in the sensor dot, we measure an SNR of 1 for an integration time of $50 \mu \mathrm{s}$. The magnitude of the signal increases quadratically with the gate lever arm to the sensor dot [28]. Based on the values in our device $\left(\alpha_{\text {sensor }}=0.24\right.$ and $\alpha_{\text {qubit }}=0.47$ ), power SNR could be increased by $16 \times$ simply by swapping the assignment of sensor and qubit. Such asymmetries in the lever arms, also reported for nominally identical devices, are due to a misalignment of $7 \pm 3 \mathrm{~nm}$ in the placement of the gates on the Si channel $[31,53]$.

Further improvements in power SNR (approximately $20 \times$ and $16 \times$, respectively) can be expected by further optimizing the resonator design to detect capacitance changes [53] and by lowering the noise floor through use of a quantum-limited amplifier [33]. Combining these methods, improvements in power SNR of 3 orders of magnitude are possible, bringing single-shot readout well within reach while simultaneously reducing the rf power used for readout to avoid limiting the minimum measurable Zeeman splitting.

These split-gate nanowire devices can be naturally scaled to produce $2 \mathrm{x} n$ arrays of corner quantum dots
[25,54] - such devices could represent a one-dimensional (1D) spin qubit array along one edge of the nanowire, where end qubits have charge sensors used for readout based on the approach presented here. 1D qubit arrays are well suited for certain quantum-simulation problems, such as a variational quantum eigensolver approach to the Hubbard model $[55,56]$. Spin shuttling [57] or qubit SWAPping [58] could transport qubits to the ends of the array, however, for some algorithms readout of an end-qubit ancilla is sufficient [59].

While it is the spin coherence time $T_{2}$ that ultimately limits qubit fidelity, the long spin-relaxation times we measure (up to $9 \mathrm{~s}$ ) are particularly encouraging for these devices. These indicate that both the CMOS-compatible fabrication methods and the nanowire geometry with its corner quantum dots are all consistent with large valley splittings and long spin-relaxation times, making them an attractive platform for scalable quantum computing.

\section{ACKNOWLEDGMENTS}

We acknowledge the financial support from the European Union's Horizon 2020 research and innovation programme under Grant Agreement No. 688539 [60]; as well as the UK's Engineering and Physical Sciences Research Council (EPSRC) through the Centre for Doctoral Training in Delivering Quantum Technologies (EP/L015242/1), QUES ${ }^{2}$ T (EP/N015118/1), and the Hub in Quantum Computing and Simulation (EP/T001062/1). V.N.C.T. acknowledges the support from the Telefónica British-Spanish society scholarship. M.F.G.Z. acknowledges support form the Royal Society Industry Fellow Programme.

\section{APPENDIX A: DEVICE DESCRIPTION}

The device is a 7-nm-tall silicon nanowire patterned from a silicon-on-insulator (SOI) substrate with a 145-nm-thick buried oxide. An omega-shape MOS gate wraps around the Si mesa nanowire, with a stack consisting of 50-nm poly-Si, 5-nm TiN, and 6-nm thermal $\mathrm{SiO}_{2} / \mathrm{Si}$. Under a suitable gate voltage, quantum dots can form separately along the top edges of the mesa nanowire. For independent control of each dot, the wrap-around gate is split along the nanowire direction using e-beam lithography forming two gates that face each other $\left(G_{\text {sensor }}\right.$ and $\left.G_{\text {qubit }}\right)$. Although these gates are nominally identical, we observe a misalignment of $7 \pm 3 \mathrm{~nm}$ in the placement of the gates on the Si channel, in which the "sensor gate" overlaps the channel less so than the other gate [53]. For further control, the silicon substrate can be used as a back gate and an overarching metal line as a top gate. These two gates modify the dot electron wave function [26,32] and, therefore, the tunnel rates between dot and reservoir. Changes in voltage applied to the metal line require stabilization for a few days, however, thereafter the new 
properties remain constant and stable for extended periods sufficient for the entire experiment.

The nanowire width, $W$, and gate length, $L_{g}$, can be engineered to achieve different interdot coupling and dot sizes, respectively. Device A has a gate length of $L_{g}=50 \mathrm{~nm}$ and a width of $W=80 \mathrm{~nm}$, whereas device B has $L_{g}=40$ $\mathrm{nm}$ and $W=70 \mathrm{~nm}$. The splitting between gates, $S_{v}$, is $S_{v}=50 \mathrm{~nm}$ for device A and $S_{v}=40 \mathrm{~nm}$ for device B.

The gates are covered by $34-\mathrm{nm}$-wide $\mathrm{Si}_{3} \mathrm{~N}_{4}$ spacers. On one hand, the spacer separates the reservoirs from the central part of the intrinsic nanowire, by protecting the intrinsic silicon from the ion implantation, which defines the reservoirs. And, on the other hand, it also covers the split between the independent gates, $G_{\text {sensor }}$ and $G_{\text {qubit }}$, since the length of both gate spacers is larger than the intergate gap.

Moreover, the nanowire region below $G_{\text {sensor }}$ is lightly Bi doped with a dose of $6 \times 10^{10}$ at $/ \mathrm{cm}^{2}$. This gives an average of approximately one Bi dopant per window of $40 \mathrm{~nm} \times 40 \mathrm{~nm}$.

\section{APPENDIX B: MEASUREMENT SETUP}

\section{Description of the dc and rf circuitry}

Measurements are performed at the base temperature of a dilution refrigerator $(15 \mathrm{mK})$. Dc voltages, $\left(V_{\text {sensor }}\right.$, $\left.V_{\text {qubit }}, V_{\text {top }}\right)$, are delivered through filtered cryogenic constantan loom. The voltage on the metal line, $V_{\text {top }}$, is kept at $-10 \mathrm{~V}$ in device $\mathrm{B}$ to reduce the qubit dot-reservoir tunneling rate. The rf signal for gate-based readout and the fast pulses are delivered through attenuated and filtered coaxial lines. The PCB contacts are connected to the device gates through on-chip aluminium bond wires. Highfrequency and de signals are combined using on-PCB bias tees. The bias tee acts on the pulses sent to the qubit gate as a high pass filter. This effect is compensated by pulse engineering using the inverse of the filter transfer function, such that after passing through the bias tee, the pulses have the desired lineshape. The resonator is formed by an $80-\mathrm{nH} \mathrm{NbN}$ planar spiral inductor [30] placed in parallel to the parasitic capacitance to ground of the PCB and the device. The PCB is made from $0.8-\mathrm{mm}$-thick RO4003C with immersion silver finish. The reflected $\mathrm{rf}$ signal is first amplified by $26 \mathrm{~dB}$ at $4 \mathrm{~K}$ (LNF-LNC0.6_2A) and further amplified at room temperature. Then, the reflected signal magnitude and phase are obtained using quadrature demodulation (Polyphase AD0540b) and measured using a digitizer (Spectrum M4i.4451-x4).

\section{Resonator}

The resonant frequency, $f_{r}$, and loaded $Q$ factor, $Q_{L}$, vary with respect to the magnetic field due to changes in the inductor kinetic inductance (see Fig. 5). The resonant frequency can be calculated as $f_{r}=1 / 2 \pi \sqrt{L\left(C_{c}+C_{0}\right)}$,
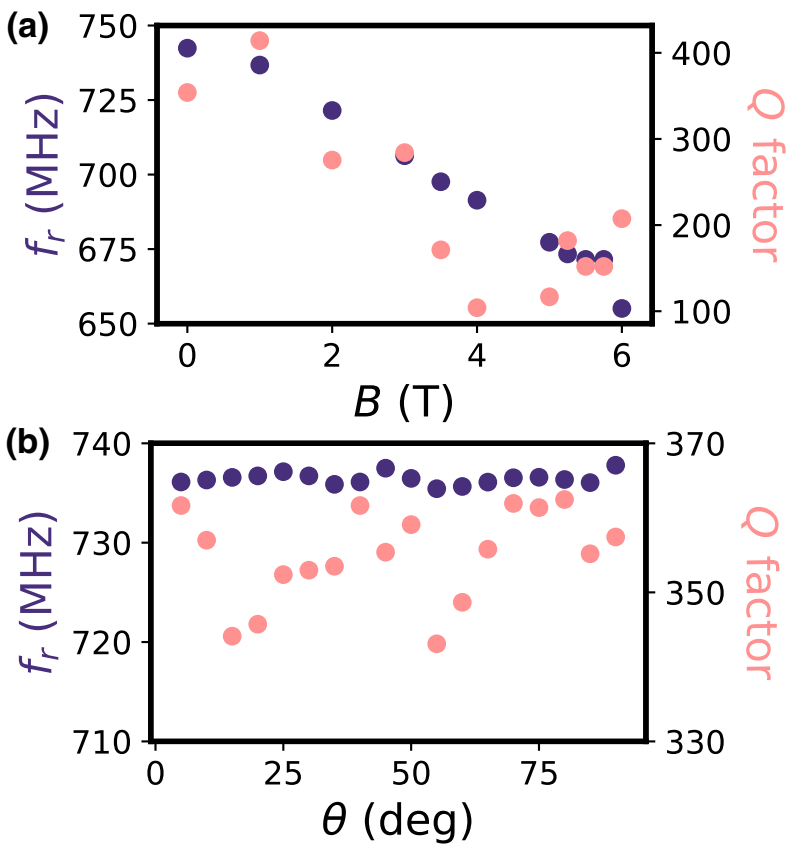

FIG. 5. (a) $Q$ factor and resonant frequency of the resonator at different magnetic fields applied in the $[\overline{1}, 1,0]$ crystallographic direction. The error bars are smaller than the scatter dots. (b) $Q$ factor and inductance when $B=1 \mathrm{~T}$ is applied in different directions. $\theta$ is the angle of the magnetic field with respect to $[\overline{1}, 1,0]$ in the plane of the device, as in Fig. 1.

where $L$ is the inductor value and $C_{0}=520 \mathrm{fF}$ is the sum of the device capacitance, $C_{d}$, and the parasitic capacitance, $C_{p} . C_{c}$, the coupling capacitance, is known and equal to $C_{c}=50 \mathrm{fF}$. The $Q$ factor can be extracted by measuring the $S$-parameter correspondent to the forward transmission, $S_{21}=20 \log _{10}|\Gamma|$, with a vector network analyser (VNA), which provides the reflection coefficient $|\Gamma|$ of the reflectometry setup. Although the resonance is undercoupled, the demodulated phase shift is still proportional to the dot capacitance shift in first order: $\Delta \phi \approx$ $-\left(2 Q_{\mathrm{int}}^{3} Z_{0} / R_{d}\right)\left[\Delta C_{d} /\left(C_{c}+C_{p}\right)\right]$, where $Q_{\text {int }}$ is the internal $Q$ factor, $Z_{0}=50 \Omega$ is the line impedance, and $R_{d}$ is the resistance seen from the dot gate to ground.

\section{APPENDIX C: READOUT OFFSET TUNING}

Observing spin-dependent tunneling requires careful tuning of the qubit-gate offset voltage, $V_{Q}$. Figures 6(a) and 6(e) show the time-dependent normalized demodulated phase at the "read" stage of the three-level sequence, for different $V_{Q}$, each averaged 1024 times. For low offsets, the electron tunnels out of the qubit dot regardless of the spin state [Fig. 6(d)], whereas for higher offset voltages it always remains in the dot [Fig. 6(b)]. At intermediate offsets, only electrons with spin up can tunnel out, producing the observed spin-dependent feature. Due to the choice of different readout points in the stability diagram (see main 
text), the rf signal for device A is maximal when an electron is present in the qubit dot, while for device B the rf signal is maximal for the empty qubit dot.

\section{Spin-readout simulations}

The averaged demodulated signal during the readout stage is proportional to the expectation number of electrons in the qubit dot [61]. We model the qubit-dot occupancy using a rate equation that considers three possible states: spin up (in the qubit dot), spin down (in the qubit dot), or no electron in the qubit dot. The ratio at which the levels are populated or emptied is given by their respective tunnel rates. The tunneling rates depend on $V_{Q}$, the electron temperature and the natural tunnel rate $\Gamma_{0}$, which we take to be spin independent. Assuming elastic tunneling and that the reservoir has a continuous energy spectrum, the dot-toreservoir tunneling rate follows a Fermi-Dirac distribution [41]:

$$
\Gamma_{\text {in (out) }}=\frac{\Gamma_{0}}{1+\exp \left[+(-) \Delta E / k_{B} T\right]} .
$$

Here, $k_{B}$ is Boltzmann's constant and $\Delta E$ is the energy difference between the relevant dot state and the lead Fermi energy. $\Delta E=|e| \alpha_{Q Q}\left(V_{\downarrow}-V_{Q}\right)$ for the spin-down state and $\Delta E=|e| \alpha_{Q Q}\left(V_{\downarrow}+E_{z}-V_{Q}\right)$ for the spin-up state, where $V_{\downarrow}$ is the voltage at which the $|\downarrow\rangle$ state and reservoir potentials align, $e$ is the electron charge, $\alpha_{Q Q}$ is the lever arm of the qubit gate on the qubit dot, and $E_{z}$ is the Zeeman energy. Therefore, four different tunneling rates can be defined: $\Gamma_{\downarrow}^{\text {in }}, \Gamma_{\downarrow}^{\text {out }}, \Gamma_{\uparrow}^{\text {in }}$, and $\Gamma_{\uparrow}^{\text {out }}$, i.e., two per dot state.

During the read stage, the sum of the probabilities of finding the electron in the dot with a spin up, $N_{\uparrow}$, with a spin down, $N_{\downarrow}$, or out of the dot, $N_{\text {out }}$, remains constant (and equal to one) such that the time-dependent derivative of the total electronic number is equal to zero: $d N_{\text {total }} / d t=$ $d N_{\text {out }} / d t+d N_{\uparrow} / d t+d N_{\downarrow} / d t=0$.

The rate equation can be summarized by the following system of differential equations:

$$
\begin{aligned}
\frac{d N_{\uparrow}}{d t} & =-\Gamma_{\uparrow}^{\text {out }} N_{\uparrow}+\Gamma_{\uparrow}^{\text {in }} N_{\text {out }}, \\
\frac{d N_{\downarrow}}{d t} & =-\Gamma_{\downarrow}^{\text {out }} N_{\downarrow}+\Gamma_{\downarrow}^{\text {in }} N_{\text {out }}, \\
\frac{d N_{\text {out }}}{d t} & =\Gamma_{\uparrow}^{\text {out }} N_{\uparrow}+\Gamma_{\downarrow}^{\text {out }} N_{\downarrow}-\left(\Gamma_{\downarrow}^{\text {in }}+\Gamma_{\uparrow}^{\text {in }}\right) N_{\text {out }} .
\end{aligned}
$$

When the system of differential equations is rewritten as a matrix, its solution has the general form:

$$
\left(\begin{array}{c}
N_{\uparrow} \\
N_{\downarrow} \\
N_{\text {out }}
\end{array}\right)=x e^{v_{1} t} \mathbf{v}_{1}+y e^{v_{2} t} \mathbf{v}_{2}+z e^{v_{3} t} \mathbf{v}_{3},
$$

where $\mathbf{v}_{1}, \mathbf{v}_{2}$, and $\mathbf{v}_{3}$ are the matrix eigenvectors and $v_{1}, v_{2}$, and $v_{3}$ their correspondent eigenvalues given by

$$
\begin{aligned}
& v_{1}=0, \\
& \left.v_{2}=\frac{1}{2}\left\{-4 \Gamma_{0}-\sqrt{\left(4 \Gamma_{0}\right)^{2}-4\left[\Gamma_{\uparrow}^{\text {in }} \Gamma_{\downarrow}^{\text {out }}+\Gamma_{\uparrow}^{\text {out }}\left(\Gamma_{\downarrow}^{\text {out }}+\Gamma_{\downarrow}^{\text {in }}\right)\right.}\right]\right\}, \\
& v_{3}=\frac{1}{2}\left\{-4 \Gamma_{0}+\sqrt{\left(4 \Gamma_{0}\right)^{2}-4\left[\Gamma_{\uparrow}^{\text {in }} \Gamma_{\downarrow}^{\text {out }}+\Gamma_{\uparrow}^{\text {out }}\left(\Gamma_{\downarrow}^{\text {out }}+\Gamma_{\downarrow}^{\text {in }}\right)\right]}\right\} .
\end{aligned}
$$

$x, y$, and $z$ are the constants determined by the initial conditions. Here, it is assumed that the qubit dot is always emptied during the empty stage and populated after the load stage such that the readout initial conditions include an electron in the dot with a random spin polarization:

$$
\begin{aligned}
& N_{\uparrow}(t=0)=1 / 2, \\
& N_{\downarrow}(t=0)=1 / 2, \\
& N_{\text {out }}(t=0)=0 .
\end{aligned}
$$

These assumptions are based on the fact that the measured tunneling times are much shorter than the duration of the pulses. The averaged demodulated phase is proportional to the expected dot occupation number, $1-N_{\text {out }}$, for device A and to $N_{\text {out }}$ for device B.

Properties of the system can be obtained by examining the behavior in particular regimes where the dynamics can be simply understood. First, at low offsets the dot state is well above the lead Fermi energy [see line-cut 2 in Fig. 6(a)]. In this regime, $\Gamma_{\downarrow}^{\text {in }}$ and $\Gamma_{\uparrow}^{\text {in }}$ tend to zero, whereas $\Gamma_{\downarrow}^{\text {out }}$ and $\Gamma_{\uparrow}^{\text {out }}$ reach their maximum value, $\Gamma_{0}$, which can thus be obtained by fitting the demodulated phase over time to an exponential decay [see Figs. 6(g) and 6(i)]. In this way, we obtain tunneling rates $\Gamma_{0, A}=0.624 \pm 0.011$ $\mathrm{MHz}$ for device $\mathrm{A}$ and $\Gamma_{0, B}=0.970 \pm 0.012 \mathrm{MHz}$ for device B.

Second, by observing the demodulated phase with respect to $V_{Q}$ after some time has passed [line-cut 1 in Fig. 6(a)], the effective temperature can be inferred. The dynamics are initially described by the complete model described in Eq. (C3), however, the effect of the negative eigenvalues fades away over time and the term $\mathbf{N}=$ $x \mathbf{v}_{1}$ dominates the dot occupation. For the given initial conditions, this steady-state term reads

$$
N_{\text {out }}(t=\infty)=\frac{\Gamma_{\uparrow}^{\text {out }} \Gamma_{\downarrow}^{\text {out }}}{\Gamma_{\uparrow}^{\text {in }} \Gamma_{\downarrow}^{\text {out }}+\Gamma_{\uparrow}^{\text {out }}\left(\Gamma_{\downarrow}^{\text {out }}+\Gamma_{\downarrow}^{\text {in }}\right)},
$$

which for the condition $E_{z} / k_{B} T \gg 1$ simplifies to $N_{\text {out }}(t=$ $\infty)=\Gamma_{\downarrow}^{\text {out }} / \Gamma_{0}$. Therefore, the demodulated phase with respect to $V_{Q}$ is fitted to $1-\Gamma_{\downarrow}^{\text {out }} / \Gamma_{0}$ for device $\mathrm{A}$ and $\Gamma_{\downarrow}^{\text {out }} / \Gamma_{0}$ for device B [see Figs. 6(f) and 6(h)]. From these fits we find an effective temperature of $0.157 \pm 0.012$ 


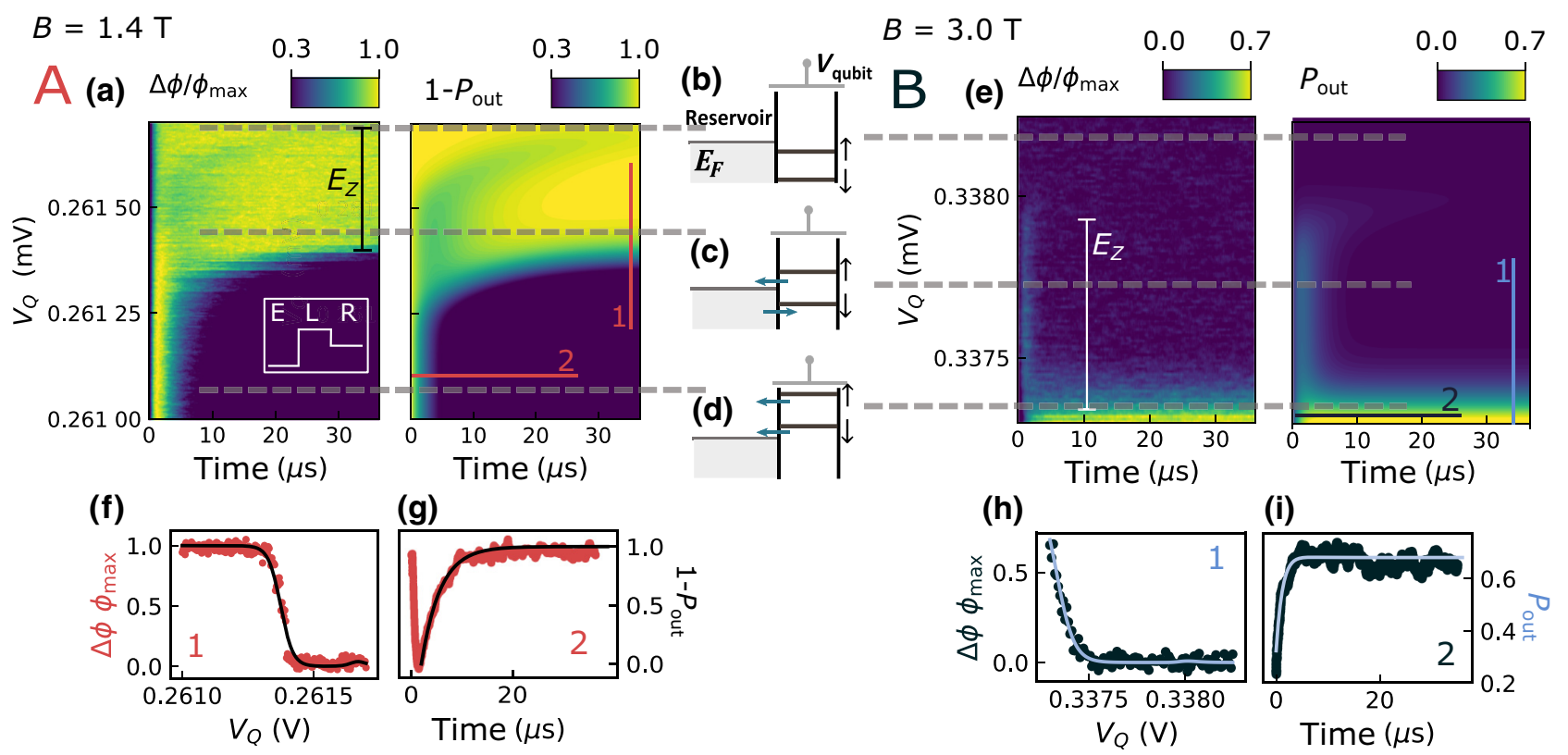

FIG. 6. (a) Left: normalized phase response of the resonator over time in the readout stage at different $V_{Q}$ offsets for device A. The pulse sequence is depicted in the inset. Right: simulation for an applied magnetic field of $1.4 \mathrm{~T}$. The simulation takes into account the mismatch between the bias-tee cutoff frequency and the compensated pulse. (b) Same for device B at $B=3 \mathrm{~T}$. (b)-(d) Diagrams of the qubit-dot electrochemical potential with respect to the lead Fermi energy at three different offsets. At offset (b) the electron remains in the dot. In (c) only spin-up electrons can tunnel out from the dot and, shortly afterwards, an electron with spin down comes back to the dot. In (d) the electron always tunnels out. (f) Dot occupation number along line 1 comparing measurement (dotted) with simulation (line). (g) Dot occupation number as a function of time at low offsets along line 2 . The phase rise time due to the resonator bandwidth corresponds to the first microsecond of the graph. (e),(h),(i) Corresponding data and simulation for device B.

$\mathrm{K}$ for device A and $0.275 \pm 0.022 \mathrm{~K}$ for device $\mathrm{B}$ (see Appendix $\mathrm{F}$ for full discussion of the origin of this effective temperature and noise sources).

The Zeeman splitting, $E_{z}$, is calculated from the width in voltage of the spin-dependent "tail" seen in Fig. 6. The Zeeman splitting is plotted for different magnetic fields in Fig. 9.

To improve the fit to the data, the simulations of device A [Figs. 6(b) and 7] include a voltage drift over time due to a cutoff-frequency miscalculation of the bias-tee high pass filter (nominally taken to be $16 \mathrm{kHz}$, but fitted to be $15.915 \mathrm{kHz}$ ). This small frequency mismatch does not affect measurements on the spin-relaxation time.

\section{APPENDIX D: TWO-LEVEL PULSE SEQUENCE (LOAD-READ ONLY)}

If the "empty" stage is removed from the pulse sequence to leave just the "load" and "read" steps, the dot remains occupied unless the electron tunnels out during the read stage. In this two-level pulse sequence, the electron in the dot eventually decays to the spin-down ground state, so that no spin-up signature is observed (see Fig. 7 for measurements and simulations). The rate equations presented in the previous section to simulate the dot occupation number remain valid in this case - only the initial conditions change. In the previous case, when the dot is initialized, the probability of finding the dot empty after reading out is $N_{\text {out }}=\Gamma_{\downarrow}^{\text {out }} / \Gamma_{0}$. For the two-level sequence, only when the dot has been emptied can a new electron be loaded (with random spin polarization). Thus, the initial conditions are

$$
\begin{aligned}
& N_{\uparrow}(t=0)=\frac{\Gamma_{\downarrow}^{\text {out }}}{2 \Gamma_{0}}, \\
& N_{\downarrow}(t=0)=1-N_{\uparrow}(t=0), \\
& N_{\text {out }}(t=0)=0,
\end{aligned}
$$

and these are used in the simulations presented in Fig. 7.

\section{APPENDIX E: GATE LEVER ARMS}

The gate lever-arm map the voltage applied to each gate to the electrostatic energy at the dot. In this system, where two quantum dots are placed in parallel, the Coulomb diamonds of each dot can be measured independently. The sensor-dot-sensor-gate lever arm $\left(\alpha_{S S}\right)$ is calculated by measuring Coulomb diamonds in the same dot-to-lead transition used for spin readout [see Fig. 8(c)]. At $V_{\text {s.d. }} \neq 0$, the transition splits in two. These two lines with slope $m_{1}$ and $m_{2}$, delimit the set of voltages at which the dot level is 


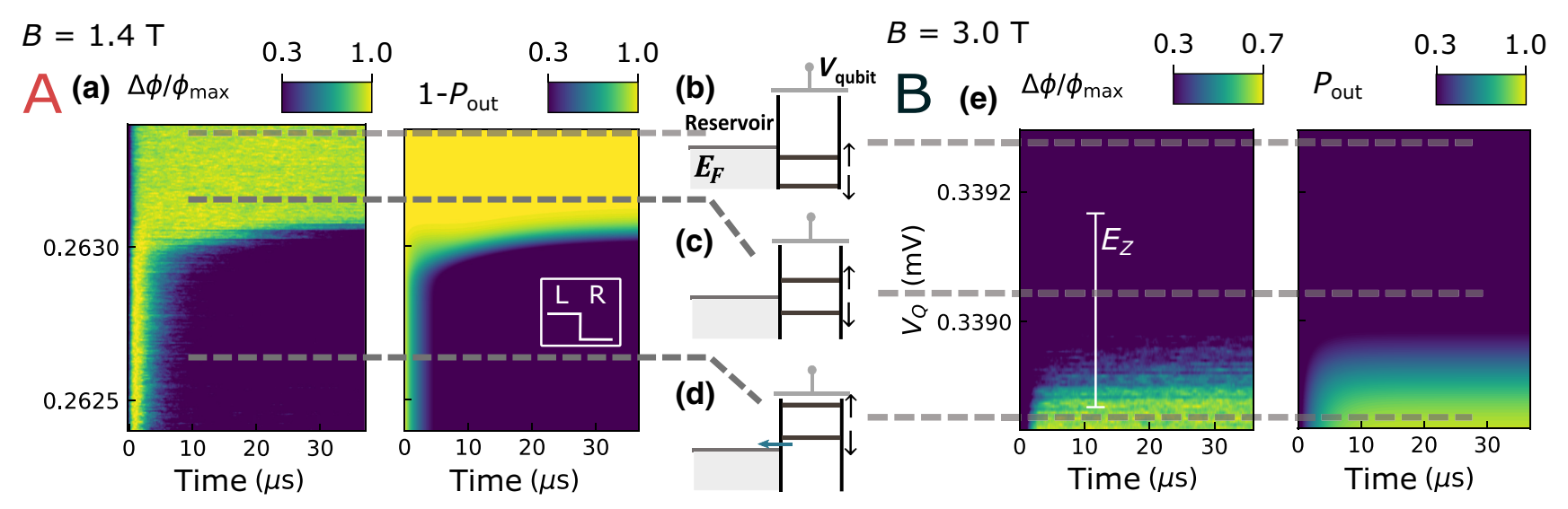

FIG. 7. Two-level pulse sequence based on "load" and "read." (a) Demodulated phase over time in the readout stage normalized at each $V_{Q}$ offset for device A (left) and its simulation (right) for an applied magnetic field of $1.4 \mathrm{~T}$. The pulse sequence is depicted in the inset. (b)-(d) Diagrams of the qubit-dot electrochemical potential with respect to the lead Fermi energy at three different pulse offsets, $V_{Q}$. (e) The same as (a), but for device B at $B=3 \mathrm{~T}$.

in the bias window and the lever arm can be calculated as the inverse of the slope difference: $\alpha_{S S}=1 /\left|1 / m_{1}-1 / m_{2}\right|$ [62]. In addition, the gate for the qubit dot can influence the sensor dot, such that in general there is a lever-arm matrix [63]:

$$
\left(\begin{array}{c}
\Delta \mu_{S} \\
\Delta \mu_{Q}
\end{array}\right)=\left(\begin{array}{cc}
\alpha_{S S} & \alpha_{S Q} \\
\alpha_{Q S} & \alpha_{Q Q}
\end{array}\right)\left(\begin{array}{c}
V_{S} \\
V_{Q}
\end{array}\right)
$$

where $\Delta \mu_{S}$ and $\Delta \mu_{Q}$ are the electrochemical potentials of the sensor and qubit dot, respectively. The effect of the cross terms is visible in the stability diagram, where the slope of dot-to-lead transitions is given by the ratio between lever arms. This way, the cross lever arm is found to be $\alpha_{S Q}=\Delta V_{S} / \Delta V_{Q} \times \alpha_{S S}$.

The qubit-dot-qubit-gate lever arm, $\alpha_{Q Q}$, is determined by a temperature study in which the qubit-dot occupation number is fitted with respect to $V_{Q}$ to a Fermi distribution, as in Figs. 6(g) or 6(i). In these traces, $V_{Q}$ is swept so the qubit-dot transitions from an empty to occupied state. The broadening in this transition can have different origins: (1) the QD level broadening due to finite lifetime, (2) the effect of the rf-carrier power on the qubit-dot electrochemical potential via the cross capacitance, or (3) the reservoir electron temperature. We focus on the latter and analysed this broadening with respect to the fridge temperature. At low temperatures, the broadening is constant and, as the temperature in the fridge is raised, it increases linearly with respect to the fridge temperature (see Fig. 9). In this way, the temperature can be related to the transition broadening as $k_{B} T_{e}=e \alpha_{Q Q} V_{Q}$ and can be fitted to $T_{e}=\sqrt{T_{0}^{2}+T_{\text {fridge }}^{2}}$ [44]. We obtain a $T_{0}=230 \pm 9 \mathrm{mK}$ and a $\alpha_{Q Q}=0.478 \pm 0.008$.

Finally, the second cross lever arm is obtained with the stability diagram as $\alpha_{S Q}=\alpha_{Q Q}\left(\Delta V_{Q} / \Delta V_{S}\right)$ using the slope of the qubit dot-to-lead transition, which, although invisible, can be deduced by joining the shifts in the sensor electronic transitions (see Fig. 8). The lever-arm values for devices A and B are summarized in Table I.

The $g$ factor is calculated using $E_{Z}=g \mu_{B} B=$ $e \alpha_{Q Q} \Delta V_{Q}$, where $\Delta V_{Q}$ is obtained from Figs. 6(a) and $6(c)$ at different magnetic fields. The calculated $g$ factor is $g=1.92 \pm 0.11$.

\section{APPENDIX F: SIGNAL OPTIMIZATION}

\section{Power dependence of the line broadening}

To differentiate between spin states and achieve spin readout, the Zeeman splitting, $E_{z}=g \mu_{B} B$ must be greater than the broadening of the $0 \rightarrow 1$ charge transition in the qubit dot depicted in Fig. 6(f). As discussed above, the broadening has at least three different sources: (1) the reservoir electron temperature, (2) the perturbations in its potential produced by the rf carrier via cross capacitance to the sensor-dot gate, and (3) the dot-state broadening due to tunneling. These three noise sources limit the minimum magnetic field at which the spin state can be accurately determined.

Here, we study how the power from the rf carrier sent to the "sensor" gate affects the "qubit"-dot potential. Using reflectometry, the linewidth of an electronic transition is set by the electronic temperature as long as the thermal energy, $k_{B} T$, is larger than the QD level broadening, $\hbar \gamma$, where $\gamma$ is the tunneling rate. When the electron temperature dominates, the parametric capacitance contribution due to the ability of the electron to tunnel in and out of the dot is proportional to $\Delta C_{d} \propto 1 / \cosh ^{2}\left(\epsilon / 2 k_{B} T\right)$, where $\epsilon$ is the quantum-dot level detuning with the reservoir. In the latter case, $\left(k_{B} T \ll h \gamma\right)$, the parametric capacitance follows a Lorentzian shape: $\Delta C_{d} \propto \hbar \gamma /\left[(\hbar \gamma)^{2}+\epsilon^{2}\right]$ [64]. At the same time, power applied to the sensor dot required for reflectometry can be a source for line broadening. 

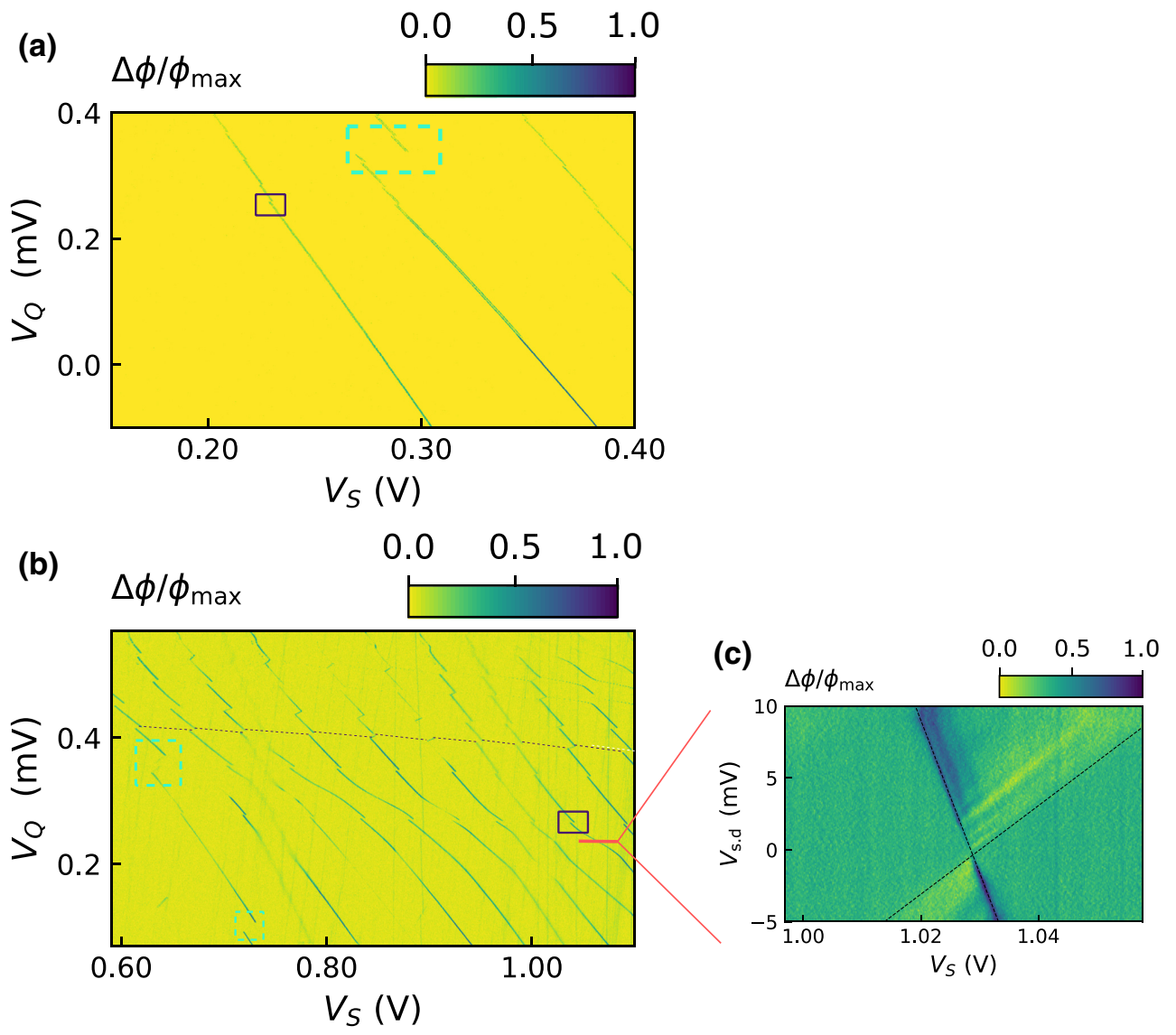

FIG. 8. Lever-arm and device characterization. (a) Device A stability diagram. A solid square indicates the readout area corresponding to the first electronic transition of the qubit dot since no other shifts are visible for a large range of smaller $V_{Q}$. The dashed square indicates dotdonor transitions. The donor is presumed to be bismuth since the sample is bismuth doped. (b) As above for device B. The black dashed line helps the eye to follow one of the qubitdot electronic transitions. (c) Coulomb diamond of the sensor dot in device B from the sensor dot-to-lead transition used in the readout. The slopes used for calculating $\alpha_{S S}$ (see text) are marked with dashed black lines.
When power broadening is dominant [see Fig. 10(a)], the linewidth increases as

$$
\epsilon_{1 / 2}=\epsilon_{(1 / 2) 0} \sqrt{1+\frac{P}{P_{0}}},
$$

where $\epsilon_{(1 / 2) 0}$ is the natural width due to electron temperature or tunneling rates and $P_{0}$ is the power at which the power starts dominating the transition width. The broadening effect of the $r f$ readout signal on the sensor potential can be translated to an effective temperature and, using the previously calculated $g$ factor, to an effective magnetic field, $B_{\text {noise }}$ (see Appendix E).

For the sensor dot, the natural width, $\epsilon_{(1 / 2) 0}$, corresponds to a tunneling rate of $4.87 \pm 0.04 \mathrm{GHz}$ or an electron temperature of $130 \pm 1 \mathrm{mK}$, consistent with previous results in Si nanowires [64].

The rf readout tone applied to the sensor gate can be transferred to the qubit dot due to the cross capacitance between dots or a direct capacitance between the (a)

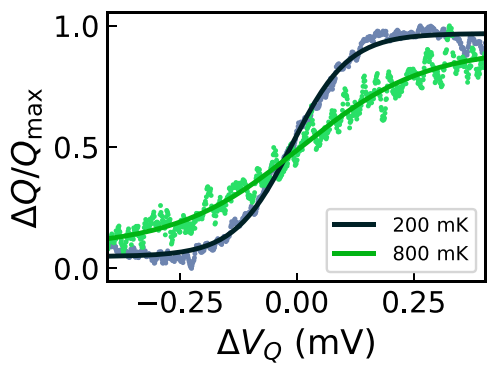

(b)

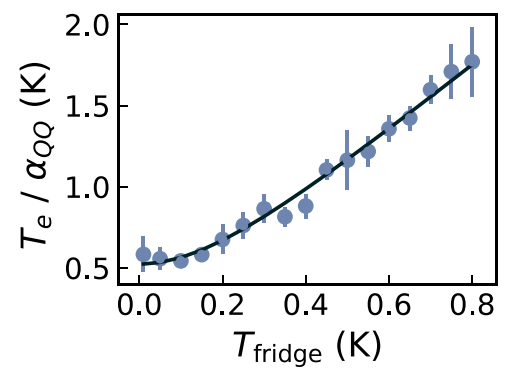

(c)

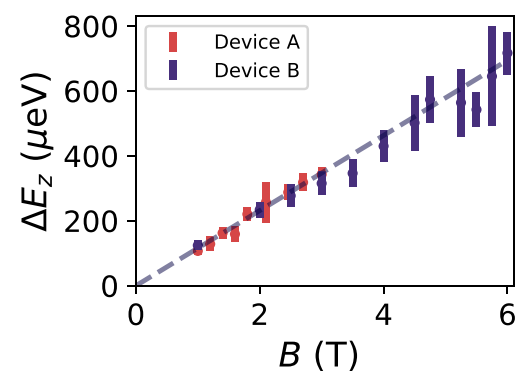

FIG. 9. Qubit-dot lever arm from temperature measurements. (a) Homodyne $I / Q$ voltage as a function of $\Delta V_{Q}$, at fridge temperatures of 200 and $800 \mathrm{mK}$ for device B. (b) Width of the Fermi-Dirac distribution measured in (a) as a function of the fridge temperature and fit. (c) Zeeman energy obtained as $\Delta E_{z}=e \alpha_{Q Q} V_{Q}$ from Fig. 6(a), where $Q Q$ is the lever arm calculated in (b). Dashed line shows the Zeeman energy for $g=2$. 
TABLE I. Lever arms. $X X$ refers to the subindex of the alpha factor, which can take the values $S S, S Q, Q Q$, or $S Q . \sigma_{\alpha_{X X}}$ refers to the standard error in the extracted values.

\begin{tabular}{lccc}
\hline \hline & $X X$ & $\alpha_{X X}$ & $\sigma_{\alpha_{X X}}$ \\
\hline Device A & QQ & 0.58 & 0.03 \\
& SQ & 0.070 & 0.006 \\
\hline Device B & SS & 0.239 & 0.004 \\
& SQ & 0.121 & 0.002 \\
& QQ & 0.478 & 0.008 \\
& QS & 0.078 & 0.004 \\
\hline \hline
\end{tabular}

sensor gate and the qubit dot: $\mu_{Q}=\left(\alpha_{Q S} / \alpha S S\right) \Delta \mu_{S}$ (see Appendix E). In Fig. 10 we compare (i) the broadening measured on the sensor dot that has been converted to an expected qubit dot broadening, with (ii) a direct measurement of the qubit dot broadening as in Fig. 9(a). We observe that increasing the rf readout power, increases the perturbation for both methods, however, they lead to a different natural width. This suggests that the predominant broadening at lower power does not come from the electron temperature, since it should be the same under both measurements, but from the tunneling rates, which are higher in the sensor dot. At higher powers, such as the one used for spin readout $(P=-83 \mathrm{dBm})$, we can deduce that the major contribution to line broadening (and thus effective temperature) comes from the rf tone used for readout. The contribution from the rf tone can be reduced by optimizing the resonator so less power is needed to show a measurable phase shift [30] and/or using cryogenic amplifiers with lower noise temperature such as a Josephson parametric amplifier [33] allowing operation at lower rf power due to a decreased noise level. Moreover, although the mutual capacitance between dots is necessary for this readout, the cross capacitance between the sensor gate and the quantum dot should be as small as possible.

\section{Visibility}

The reflectometry signal is optimized by selecting the power and qubit offset voltage that gives the highest visibility of the spin-up fraction. Figure 10(b) shows a comparison of the spin-up fraction at different qubit-dot offset voltages and rf power applied. The spin-up signature is more visible at higher voltages up to a point where the power broadening counteracts the increment in the signal.

\section{APPENDIX G: EXCITED STATE SPECTROSCOPY}

The main text describes five different regimes of interest $(\mathrm{I}-\mathrm{V})$ in the context of excited-state spectroscopy. In regimes II-V, the probability of finding an electron with
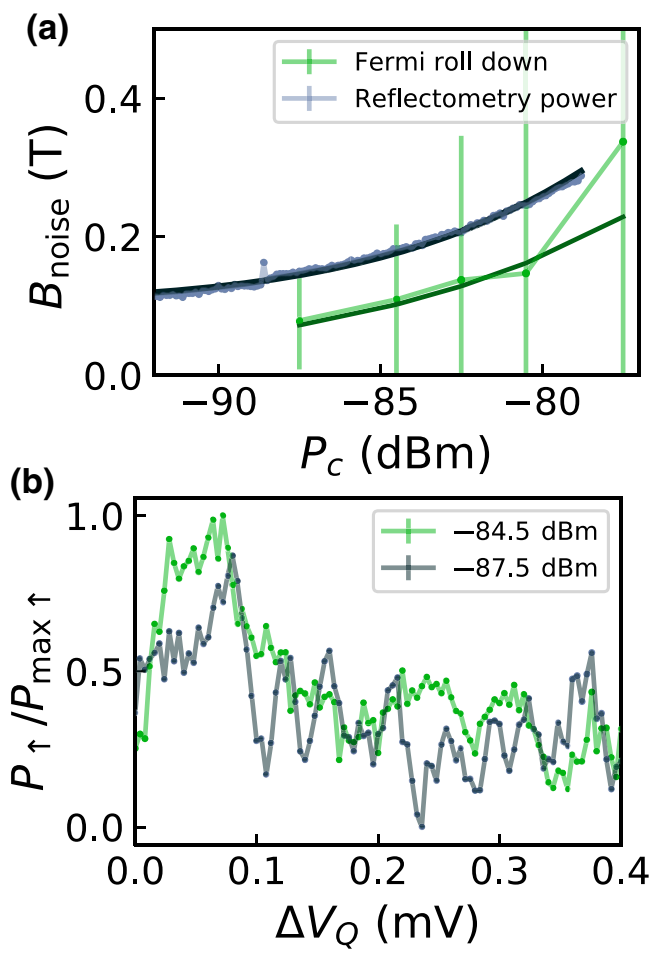

FIG. 10. Perturbation of the qubit-dot electrochemical potential, given in Tesla, due to the rf-carrier power, $P_{c}$. (a) Blue: electronic transition half width maximum, $\epsilon_{1 / 2}$ of the sensor dot, converted to a qubit-dot potential using the cross lever arm $\alpha_{Q S}$. Error bars are smaller than the dot size. Green: a direct measurement of broadening obtained by sweeping the qubit-dot voltage [see Fig. 9(a)]. (b) Spin-up fraction at different readout offsets, $V_{Q}$, and powers at $1 \mathrm{~T}$.

spin up at different load voltages $V_{Q, L}$ is argued to be

$$
P \uparrow\left(V_{Q, L}\right)=\frac{\Gamma_{\text {load }, g \uparrow}+\Gamma_{\text {load }, e \uparrow}}{\Gamma_{\text {load }, g \uparrow}+\Gamma_{\text {load }, e \uparrow}+\Gamma_{\text {load }, g \downarrow}+\Gamma_{\text {load }, e \downarrow}} .
$$

The parameters obtained from fitting the data to the expression above are summarized in Table II. Here, $E_{V}$ is the valley splitting, $T_{g}$ is the effective temperature for the ground state, $T_{e}$ is the effective temperature for the excited state, and $A_{1}=\Gamma_{0, g} / \Gamma_{0, e}$ is the ratio between the excitedand the ground-state natural-tunneling rates. The effective temperature of the excited states is left as a fitting parameter to include effects arising from the finite excited-state lifetime. In contrast, any lifetime broadening of $\left|g_{\uparrow}\right\rangle$ is neglected based on the long measured $T_{1}$ times $(>1 \mathrm{~ms})$.

\section{APPENDIX H: $T_{1}$ FITTING PROCEDURE}

The relaxation time, $T_{1}$, describes the time constant at which the spin-up decays to the spin-down state. Once an electron is loaded to the dot, the probability of finding a spin-up state decreases exponentially with 
TABLE II. Fitting parameters extracted from excited-state spectroscopy.

\begin{tabular}{lcc}
\hline \hline Device A & $\boldsymbol{E}_{\mathbf{V}}$ & $681 \pm 23 \mu \mathrm{eV}$ \\
& $\boldsymbol{T}_{\mathbf{g}}$ & $370 \pm 200 \mathrm{mK}$ \\
& $\boldsymbol{T}_{\mathbf{e}}$ & $510 \pm 160 \mathrm{mK}$ \\
& $\boldsymbol{A}_{\mathbf{1}}$ & $2.0 \pm 0.3$ \\
\hline Device B & $\boldsymbol{E}_{\mathbf{V}}$ & $571 \pm 27 \mu \mathrm{eV}$ \\
& $\boldsymbol{T}_{\mathbf{g}}$ & $300 \pm 30 \mathrm{mK}$ \\
& $\boldsymbol{T}_{\mathbf{e}}$ & $710 \pm 200 \mathrm{mK}$ \\
& $\boldsymbol{A}_{\mathbf{1}}$ & $7.7 \pm 0.9$ \\
\hline \hline
\end{tabular}

respect to the time waited before reading its state following $P_{\uparrow}\left(t_{\text {wait }}\right) / P_{\uparrow}(0)=e^{-\left(t_{\text {wait }} / T_{1}\right)}$. Figures $11(\mathrm{a})$ and $11(\mathrm{~b})$ show the exponential fitting for several magnetic fields in device A and device B. We also test whether the rf carrier does not affect the relaxation during the "wait" period. Figure 11(c) shows a comparison of the relaxation times measured in device B when the rf readout tone remains on throughout the three-level pulse (purple) versus switching off the readout signal during the waiting time (red). The similar values can be explained because the rf voltage only introduces an effective level broadening corresponding with a voltage drive at the frequency of the rf carrier (742 MHz). Although this broadening is measured as an effective temperature (see Appendix F), it is unrelated to the bath electron temperature and unlikely to affect the spin of the electron, with a Zeeman splitting larger than $28 \mathrm{GHz}$ $(B=1 \mathrm{~T})$.

\section{APPENDIX I: NATURE OF THE EXCITED STATE}

In this section, we discuss the possibility that the excited state probed in the devices is in fact an orbital rather than a valley excitation. If so, the valley excitation must lie at low energy $(\lesssim 110 \mu \mathrm{eV}$ in device $\mathrm{A}$ and $\lesssim 280 \mu \mathrm{eV}$ in device B), below the lowest magnetic field explored in the experiments since no signs of such a valley state are found in measurements like the one shown in Fig. 4(a). Although very small valley splittings have indeed been measured in specific devices [38], larger valley splittings, closer to the excited-state energies we measure, would be more consistent with recent measurements in the few electrons regime [3]. Furthermore, according to effective mass calculations, orbital excitations would rather be expected in the few meV range [65], larger than the values we measure. Therefore, an orbital excitation cannot be ruled out, but is definitely not the most likely assumption. Nonetheless, the main conclusions of this work and discussion would be little affected in principle. The experiments indeed probe the spin degree of freedom as long as the Fermi level of the contacts lies between the pair of spin-up and the pair of spin-down valley states at readout; also, as valley relaxation is presumably much faster than spin relaxation, and little dependent on the magnetic field orientation, the angular dependence of the relaxation time shall be globally the same as discussed below.

\section{APPENDIX J: DEPENDENCE OF SPIN RELAXATION $\left(T_{1}\right)$ ON MAGNETIC FIELD}

\section{Dependence on magnetic field orientation}

We discuss here the angular dependence of the spinrelaxation rate in the $Z$ valleys of silicon. We consider a silicon quantum dot under a finite magnetic field $\mathbf{B}$. We note $|n, \sigma\rangle$ and $E_{n, \sigma}=E_{n} \pm \frac{1}{2} g_{0} \mu_{B} B \sigma$ the eigenstates and eigenenergies of the dot in the absence of spin-orbit coupling, with $\sigma= \pm 1$ the spin quantized along the magnetic field axis, $g_{0}$ the bare gyromagnetic factor of the electron and $\mu_{B}$ Bohr's magneton. In these assumptions, the orbitals $\varphi_{n}(\mathbf{r})=\langle\mathbf{r} \mid n\rangle$ can be chosen real at $\mathbf{B}=\mathbf{0}$.

In the Fermi golden rule approximation, the relaxation rate between states $|\mathbb{O}\rangle \equiv|0,-1\rangle$ and $|\mathbb{1}\rangle \equiv|0,+1\rangle$ is typically proportional to the squared matrix element(s) $|\langle\mathbb{1}|O| 0\rangle|^{2}$ of one or more observable(s) $O[36,37,46]$. We assume that $O$ is invariant under time-reversal symmetry, and does not couple spins directly $[O$ is, e.g., a local potential $V(\mathbf{r})$ (Johnson-Nyquist noise), an electric dipole operator $x, y$, or $z$ (phonons), etc...]. There must, therefore, be a mechanism such as spin-orbit coupling mixing spins in $|\mathbb{O}\rangle$ and $|\mathbb{1}\rangle$ in order to achieve nonzero $\langle\mathbb{1}|O| \mathbb{O}\rangle$ 's.

Since SOC is weak in the conduction band of silicon, we can deal with it to first order in perturbation. Let $H_{\mathrm{so}}$ be the SOC Hamiltonian. The first-order $|\mathbb{O}\rangle$ and $|\mathbb{1}\rangle$ states read

$$
\begin{aligned}
|\tilde{\mathbb{O}}\rangle= & |\mathbb{O}\rangle+\sum_{n \neq 0} \frac{\left\langle n,-1\left|H_{\mathrm{so}}\right| 0,-1\right\rangle}{E_{0}-E_{n}}|n,-1\rangle \\
& +\sum_{n} \frac{\left\langle n,+1\left|H_{\mathrm{so}}\right| 0,-1\right\rangle}{E_{0}-E_{n}-g_{0} \mu_{B} B}|n,+1\rangle, \\
|\tilde{\mathbb{1}}\rangle= & |\mathbb{1}\rangle+\sum_{n \neq 0} \frac{\left\langle n,+1\left|H_{\mathrm{so}}\right| 0,+1\right\rangle}{E_{0}-E_{n}}|n,+1\rangle \\
& +\sum_{n} \frac{\left\langle n,-1\left|H_{\mathrm{so}}\right| 0,+1\right\rangle}{E_{0}-E_{n}+g_{0} \mu_{B} B}|n,-1\rangle .
\end{aligned}
$$

Hence, since $O$ only couples same spins,

$$
\begin{aligned}
\langle\tilde{\mathbb{1}}|O| \tilde{\mathbb{O}}\rangle= & \sum_{n} \frac{\langle 0,+1|O| n,+1\rangle\left\langle n,+1\left|H_{\mathrm{so}}\right| 0,-1\right\rangle}{E_{0}-E_{n}-g_{0} \mu_{B} B} \\
& +\sum_{n} \frac{\left\langle 0,+1\left|H_{\mathrm{so}}\right| n,-1\right\rangle\langle n,-1|O| 0,-1\rangle}{E_{0}-E_{n}+g_{0} \mu_{B} B} .
\end{aligned}
$$

We further develop this expression to first order in $\mathbf{B}$, assuming $g_{0} \mu_{B} B \ll E_{1}-E_{0}$. Neglecting the action of the vector potential on the orbital motion of the electrons in 
(a)

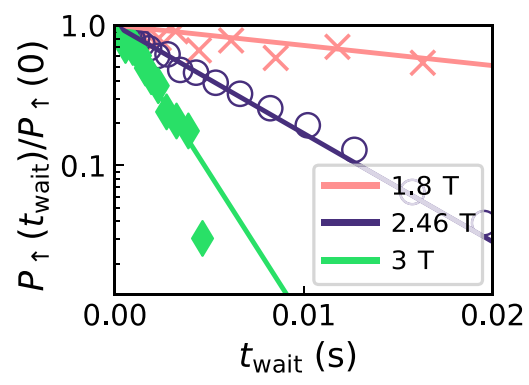

(b)

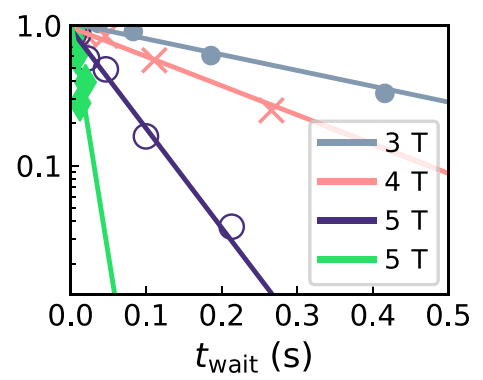

(c)

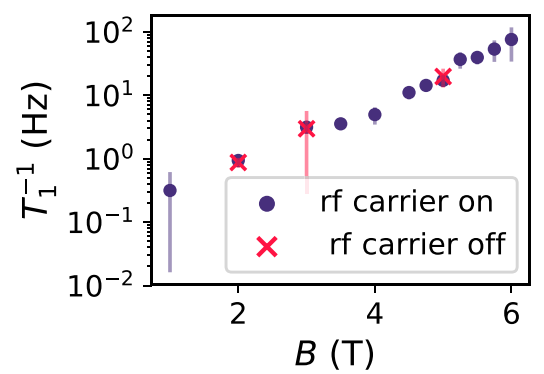

FIG. 11. (a),(b) The normalized spin-up fraction with respect to the waiting time for devices A and B, respectively, fitted to an exponential decay for different magnetic fields. (c) Comparison between the relaxation time measured with the rf-readout tone continuously on, or switched off during the waiting time.

a first place, the only $B$-dependent terms are the Zeeman energies on the denominators:

$$
\frac{1}{E_{0}-E_{n} \pm g_{0} \mu_{B} B}=\frac{1}{E_{0}-E_{n}} \mp \frac{g_{0} \mu_{B} B}{\left(E_{0}-E_{n}\right)^{2}} .
$$

Then, making use of the time-reversal symmetry relations:

$$
\begin{aligned}
\langle 0,+1|O| n,+1\rangle & =\langle 0,-1|O| n,-1\rangle^{*}=\langle n,-1|O| 0,-1\rangle, \\
\left\langle n,+1\left|H_{\mathrm{so}}\right| 0,-1\right\rangle & =-\left\langle n,-1\left|H_{\mathrm{so}}\right| 0,+1\right\rangle^{*}, \\
& =-\left\langle 0,+1\left|H_{\mathrm{so}}\right| n,-1\right\rangle,
\end{aligned}
$$

we get

$$
\langle\tilde{\mathbb{1}}|O| \tilde{\mathbb{O}}\rangle=2 g_{0} \mu_{B} B \sum_{n} \frac{\langle 0,+1|O| n,+1\rangle\left\langle n,+1\left|H_{\mathrm{so}}\right| 0,-1\right\rangle}{\left(E_{n}-E_{0}\right)^{2}} .
$$

With a SOC operator of the form $H_{\text {so }}=\sum_{k} P_{k} \sigma_{k}$, where $P_{k}$ are real-space operators (e.g., velocity operators) and $\sigma_{k}$ are the Pauli matrices (for a spin quantized along the reference axis $z$ ),

$$
\begin{aligned}
\langle\tilde{\mathbb{1}}|O| \tilde{\mathbb{O}}\rangle= & i B\left(\alpha_{x}\left\langle+1\left|\sigma_{x}\right|-1\right\rangle+\alpha_{y}\left\langle+1\left|\sigma_{y}\right|-1\right\rangle\right. \\
& \left.+\alpha_{z}\left\langle+1\left|\sigma_{z}\right|-1\right\rangle\right),
\end{aligned}
$$

where the $\alpha_{i}$ 's depend on the orbital motion of the electrons. As expected, the matrix elements $\langle\tilde{\mathbb{1}}|O| \tilde{\mathbb{O}}\rangle$ are proportional to $B$, since time-reversal symmetry must be broken by the magnetic field for $O$ to couple opposite spin states.

The orbitals $\varphi_{n}(\mathbf{r})$ being real, the matrix elements of the $P_{k}$ 's must be imaginary and those of $O$ must be real according to the time-reversal symmetry relations, Eqs. (J4b) (this is obvious if the $P_{k}$ 's are linear combinations of velocity operators and $O$ is one of the examples given above).
Therefore, $\alpha_{x}, \alpha_{y}$, and $\alpha_{z}$ are real, and

$$
\langle\tilde{\mathbb{1}}|O| \tilde{\mathbb{O}}\rangle=i B\langle+1|\alpha \cdot \sigma|-1\rangle=i B|\alpha|\left\langle+1\left|\sigma_{\hat{\alpha}}\right|-1\right\rangle,
$$

where $\sigma_{\hat{\alpha}}$ is the spin along axis $\alpha=\left(\alpha_{x}, \alpha_{y}, \alpha_{z}\right)$. Since $|-1\rangle$ and $|+1\rangle$ are defined with respect to the magnetic field axis, $\left|\left\langle+1\left|\sigma_{\hat{\alpha}}\right|-1\right\rangle\right|=\left|\sin \theta_{\alpha}\right|$, where $\theta_{\alpha}$ is the angle between the magnetic field and the vector $\alpha$. Hence,

$$
|\langle\tilde{\mathbb{1}}|O| \tilde{\mathbb{D}}\rangle|^{2} \propto \sin ^{2} \theta_{\alpha} .
$$

This gives rise to the simple uniaxial dependence measured for example in Ref. [66]. In that reference, the effects of SOC are dominated by "spin-valley" mixing, that is by the $n=1$ term in Eq. (J5) (same orbital in the other valley). In an ideal corner dot with a (110) mirror symmetry plane, $\theta_{\alpha}$ shall be the angle with the [110] axis.

The above considerations may not, however, apply when the action of the vector potential is taken into account. Indeed, in the presence of a vector potential, timereversal symmetry transforms $\varphi_{n}(\mathbf{B}, \mathbf{r})$ into $\varphi_{n}^{*}(-\mathbf{B}, \mathbf{r})$, breaking Eqs. (J4b) and the resulting cancellations. This is not expected to make much difference for spin-valley mixing as the ground states of both valleys effectively behave as zero (or, more generally, identical) angularmomentum states and are, therefore, weakly coupled by the vector potential. Yet the effects of the vector potential may become relevant when spin-valley mixing is not dominant.

In order to go further, we can write Eq. (J2) under the form:

$$
\langle\tilde{\mathbb{1}}|O| \tilde{\mathbb{O}}\rangle=\left\langle+1\left|H_{c}\right|-1\right\rangle,
$$

where the effective Hamiltonian $H_{c}$ is

$$
H_{c}=\sum_{n, k}\left(\frac{\langle 0|O| n\rangle\left\langle n\left|P_{k}\right| 0\right\rangle}{E_{0}-E_{n}-g_{0} \mu_{B} B}+\frac{\left\langle 0\left|P_{k}\right| n\right\rangle\langle n|O| 0\rangle}{E_{0}-E_{n}+g_{0} \mu_{B} B}\right) \sigma_{k},
$$


then expand $H_{c}$ to first order in $\mathbf{B}$ (being understood that $E_{n},|n\rangle$ and possibly the $P_{k}$ operators depend on $\mathbf{B}$ ):

$$
H_{c}=i B \alpha \cdot \sigma+\sum_{i, j} \lambda_{i j} B_{i} \sigma_{j}
$$

where $\lambda_{i j}$ are real scalars. Symmetry considerations may put constraints on the $\lambda_{i j}$ 's.

Assuming $\mathbf{B}=B(\cos \theta, \sin \theta, 0)$, we may then sort out the angular dependence of the matrix element $\left\langle+1\left|H_{c}\right|-1\right\rangle$. The $|+1\rangle$ and $|-1\rangle$ spin states are the eigenstates of the Zeeman Hamiltonian:

$$
H_{z}=\frac{1}{2} g_{0} \mu_{B} B\left(\cos \theta \sigma_{x}+\sin \theta \sigma_{y}\right)=\frac{1}{2} g_{0} \mu_{B} B\left[\begin{array}{cc}
0 & e^{-i \theta} \\
e^{i \theta} & 0
\end{array}\right] .
$$

Hence,

$$
\begin{aligned}
& |-1\rangle=\frac{e^{i \pi / 4}}{\sqrt{2}}\left(e^{-i \theta / 2}|\uparrow\rangle-e^{i \theta / 2}|\downarrow\rangle\right), \\
& |+1\rangle=\frac{e^{-i \pi / 4}}{\sqrt{2}}\left(e^{-i \theta / 2}|\uparrow\rangle+e^{i \theta / 2}|\downarrow\rangle\right) .
\end{aligned}
$$

The above phase factors are chosen for convenience. Then,

$$
\begin{aligned}
& \left\langle+1\left|\sigma_{x}\right|-1\right\rangle=+\sin \theta, \\
& \left\langle+1\left|\sigma_{y}\right|-1\right\rangle=-\cos \theta, \\
& \left\langle+1\left|\sigma_{z}\right|-1\right\rangle=i .
\end{aligned}
$$

Therefore, after substitution in Eq. (J9) and trigonometric manipulations,

$$
\begin{aligned}
\left\langle+1\left|H_{c}\right|-1\right\rangle= & B\left(a_{0}+i c_{1} \cos \theta+i s_{1} \sin \theta\right. \\
& \left.+c_{2} \cos 2 \theta+s_{2} \sin 2 \theta\right),
\end{aligned}
$$

where $a_{0}, c_{1}, s_{1}, c_{2}$, and $s_{2}$ are real. This matrix element does, therefore, feature $\sin n \theta$ and $\cos n \theta$ harmonics up to $n=2$. Hence the relaxation rate, which is $\propto\left|\left\langle\mathbb{1}\left|H_{c}\right| \mathbb{O}\right\rangle\right|^{2}$, features $n=0, n=2$, and $n=4$ harmonics. We may thus write, in general,

$$
\Gamma=\gamma_{0}+\gamma_{2} \cos \left[2\left(\theta-\theta_{2}^{0}\right)\right]+\gamma_{4} \cos \left[4\left(\theta-\theta_{4}^{0}\right)\right] .
$$

Note that the relaxation rate is invariant under the transformation $\theta \rightarrow \theta+\pi(\mathbf{B} \rightarrow-\mathbf{B})$, as expected. Competing relaxation mechanisms may yield different $\gamma$ 's and $\theta^{0}$ 's; yet trigonometric relations easily show that the sum over mechanisms can always be refactored under that form.

Higher-order harmonics may result from the breakdown of one of the above assumptions [first-order developments in $B$ and $H_{\text {so }}$, validity of Fermi golden rule (multiphonon and photon processes)... ], or from extrinsic contributions.
Also, the prefactors of the relaxation rates scale as a power of the Larmor frequency, $\omega\left(\omega^{3}\right.$ to $\omega^{5}$ for phonons, $\omega$ for Johnson-Nyquist noise), which may introduce extra angular dependences through the anisotropy of the $g$ factors. However, the contribution of $g$ factors to the angular dependence of the relaxation rates is presumably very weak in silicon, as they remain usually very close to 2 whatever the orientation of the magnetic field.

Examples of pure $\cos \left[4\left(\theta-\theta_{4}^{0}\right)\right]$ dependences have, for example, been given in Ref. [34] (relaxation owing to phonon-induced shear strains in a highly symmetric dot). The enumeration of possible symmetry invariants in Eq. (J11) suggests that the relative weight of $n=4$ harmonics shall actually increase when the dot gets more symmetric [going, e.g., from a single mirror symmetry plane $\left(C_{s}\right.$ group) to a double mirror symmetry plane ( $C_{2 v}$ group)].

In the present experiments, the angular dependence of the relaxation rate is indeed dominated by $n=2$ and $n=4$ harmonics, although significant $n=6$ and $n=8$ contributions may also be needed to reproduce the behavior around $\theta=0$. Without further knowledge about the shape of that particular dot, it remains, however, difficult to make detailed predictions. Still, the presence of strong $n=4$ harmonics suggests, as discussed above, that the relaxation is not dominated by spin-valley mixing at $B=1 \mathrm{~T}$ (nor at any field given the absence of measurable hot spot at the crossing between the ground valley spin-up state and the excited valley spin-down state). Both the weakness of spin-valley mixing effects and the presence of sizable $n>2$ harmonics are consistent with a dot showing high in-plane symmetry [38].

\section{Dependence on magnetic field strength}

The angular dependence described above is consistent with a small spin-valley mixing, which also leads to a weak or absent hot spot in the relaxation rate when the Zeeman splitting approaches the valley splitting. We observe that the relaxation rate increases with the magnetic field, following the predicted behavior when far from any anticrossing with higher-lying excited states [35]. Spin relaxation comes primarily from the spin-orbit interaction, which couples the spin degree of freedom with electrical noise. The electrical noise can have different sources, for example, the Johnson-Nyquist noise, which gives a contribution proportional to $c_{J} B^{3}$ [46]. In silicon, phonons can also create an electrical disturbance by deforming the lattice inhomogeneously [67], which, due to the nonzero dipole matrix elements between the dot levels, leads to spin relaxation. The leading contribution to this mechanism in the crystallographic direction [110] scales as $c_{\mathrm{ph}} B^{7}[36]$.

We fit the relaxation-rate field dependence to the general expression $T_{1}^{-1}=c_{\mathrm{ph}} B^{7}+c_{J} B^{3}$, obtaining the coefficients summarized in Table III. The large uncertainties are due to the high correlation between the two terms. 
TABLE III. Fitting parameters for the relaxation-rate magnetic field dependence.

\begin{tabular}{lcc}
\hline \hline Device A & $c_{J}$ & $4.1 \pm 0.5 \mathrm{~Hz} / T^{3}$ \\
& $c_{\mathrm{ph}}$ & $0.171 \pm 0.018 \mathrm{~Hz} / T^{7}$ \\
\hline Device B & $c_{J}$ & $0.089 \pm 0.012 \mathrm{~Hz} / T^{3}$ \\
& $c_{\mathrm{ph}}$ & $(10 \pm 4) \times 10^{-5} \mathrm{~Hz} / T^{7}$ \\
\hline \hline
\end{tabular}

[1] D. Loss and D.P. DiVincenzo, Quantum computation with quantum dots, Phys. Rev. A 57, 120 (1998).

[2] J. Yoneda, K. Takeda, T. Otsuka, T. Nakajima, M. R. Delbecq, G. Allison, T. Honda, T. Kodera, S. Oda, Y. Hoshi, N. Usami, K. M. Itoh, and S. Tarucha, A quantum-dot spin qubit with coherence limited by charge noise and fidelity higher than 99.9\%, Nat. Nanotechnol. 13, 102 (2018).

[3] M. Urdampilleta, D. J. Niegemann, E. Chanrion, B. Jadot, C. Spence, P. A. Mortemousque, C. Bäuerle, L. Hutin, B. Bertrand, S. Barraud, R. Maurand, M. Sanquer, X. Jehl, S. De Franceschi, M. Vinet, and T. Meunier, Gate-based high fidelity spin readout in a CMOS device, Nat. Nanotechnol. 14, 737 (2019).

[4] E. J. Connors, J. J. Nelson, and J. M. Nichol, Rapid HighFidelity Spin-State Readout in Si/Si-Ge Quantum Dots via rf Reflectometry, Phys. Rev. Appl. 13, 024019 (2020).

[5] J. Yoneda, K. Takeda, A. Noiri, T. Nakajima, S. Li, J. Kamioka, T. Kodera, and S. Tarucha, Quantum nondemolition readout of an electron spin in silicon, Nat. Commun. 11, 1144 (2020).

[6] M. Veldhorst, J. C. C. Hwang, C. H. Yang, A. W. Leenstra, B. De Ronde, J. P. Dehollain, J. T. Muhonen, F. E. Hudson, K. M. Itoh, A. Morello, and A. S. Dzurak, An addressable quantum dot qubit with fault-tolerant control-fidelity, Nat. Nanotechnol. 9, 981 (2014).

[7] E. Kawakami, P. Scarlino, D. R. Ward, F. R. Braakman, D. E. Savage, M. G. Lagally, M. Friesen, S. N. Coppersmith, M. A. Eriksson, and L. M. K. Vandersypen, Electrical control of a long-lived spin qubit in a $\mathrm{Si} / \mathrm{SiGe}$ quantum dot, Nat. Nanotechnol. 9, 666 (2014).

[8] M. Veldhorst, C. H. Yang, J. C. C. Hwang, W. Huang, J. P. Dehollain, J. T. Muhonen, S. Simmons, A. Laucht, F. E. Hudson, K. M. Itoh, A. Morello, and A. S. Dzurak, A twoqubit logic gate in silicon, Nature 526, 410 (2015).

[9] D. M. Zajac, A. J. Sigillito, M. Russ, F. Borjans, J. M. Taylor, G. Burkard, and J. R. Petta, Resonantly driven CNOT gate for electron spins, Science 359, 439 (2018).

[10] T. F. Watson, S. G. Philips, E. Kawakami, D. R. Ward, P. Scarlino, M. Veldhorst, D. E. Savage, M. G. Lagally, M. Friesen, S. N. Coppersmith, M. A. Eriksson, and L. M. K Vandersypen, A programmable two-qubit quantum processor in silicon, Nature 555, 633 (2018).

[11] X. Xue, T. F. Watson, J. Helsen, D. R. Ward, D. E. Savage, M. G. Lagally, S. N. Coppersmith, M. A. Eriksson, S. Wehner, and L. M. K Vandersypen, Benchmarking Gate Fidelities in a Si/SiGe Two-Qubit Device, Phys. Rev. X 9, 021011 (2019).

[12] R. Maurand, X. Jehl, D. Kotekar-Patil, A. Corna, H. Bohuslavskyi, R. Laviéville, L. Hutin, S. Barraud, M.
Vinet, M. Sanquer, and S. De Franceschi, A CMOS silicon spin qubit, Nat. Commun. 7, 13575 (2016).

[13] K. Ono, D. Austing, Y. Tokura, and S. Tarucha, Current rectification by pauli exclusion in a weakly coupled double quantum dot system, Science 297, 1313 (2002).

[14] J. M. Elzerman, R. Hanson, L. H. Van Beveren, B. Witkamp, L. M. K Vandersypen, and L. P. Kouwenhoven, Single-shot read-out of an individual electron spin in a quantum dot, Nature 430, 431 (2004).

[15] A. West, B. Hensen, A. Jouan, T. Tanttu, C. H. Yang, A. Rossi, M. F. Gonzalez-Zalba, F. E. Hudson, A. Morello, D. J. Reilly, and A. S. Dzurak, Gate-based single-shot readout of spins in silicon, Nat. Nanotechnol. 14, 437 (2019).

[16] G. Zheng, N. Samkharadze, M. L. Noordam, N. Kalhor, D. Brousse, A. Sammak, G. Scappucci, and L. M. K. Vandersypen, Rapid gate-based spin read-out in silicon using an on-chip resonator, Nat. Nanotechnol. 14, 742 (2019).

[17] P. Pakkiam, A. V. Timofeev, M. G. House, M. R. Hogg, T. Kobayashi, M. Koch, S. Rogge, and M. Y. Simmons, Single-Shot Single-Gate rf Spin Readout in Silicon, Phys. Rev. X 8, 41032 (2018).

[18] A. Morello, J. J. Pla, F. A. Zwanenburg, K. W. Chan, K. Y. Tan, H. Huebl, M. Mottonen, C. D. Nugroho, C. Yang, J. A. Van Donkelaar, A. D. Alves, D. N. Jamieson, C. C. Escott, L. C. Hollenberg, R. G. Clark, and A. S. Dzurak, Singleshot readout of an electron spin in silicon, Nature 467, 687 (2010).

[19] T. F. Watson, B. Weber, M. G. House, H. Büch, and M. Y. Simmons, High-Fidelity Rapid Initialization and Read-Out of an Electron Spin via the Single Donor D-Charge State, Phys. Rev. Lett. 115, 166806 (2015).

[20] M. Fogarty, K. Chan, B. Hensen, W. Huang, T. Tanttu, C. Yang, A. Laucht, M. Veldhorst, F. E. Hudson, K. M. Itoh, et al., Integrated silicon qubit platform with single-spin addressability, exchange control and single-shot singlettriplet readout, Nat. Commun. 9, 4370 (2018).

[21] R. Zhao, T. Tanttu, K. Y. Tan, B. Hensen, K. W. Chan, J. C. C. Hwang, R. Leon, C. H. Yang, W. Gilbert, F. E. Hudson, et al., Single-spin qubits in isotopically enriched silicon at low magnetic field, Nat. Commun. 10, 5500 (2019).

[22] P. Harvey-Collard, B. D’Anjou, M. Rudolph, N. T. Jacobson, J. Dominguez, G. A. TenEyck, J. R. Wendt, T. Pluym, M. P. Lilly, W. A. Coish, M. Pioro-Ladriere, and M. S. Carroll, High-Fidelity Single-Shot Readout for a Spin Qubit via an Enhanced Latching Mechanism, Phys. Rev. X 8, 021046 (2018).

[23] M. J. Curry, M. Rudolph, T. England, A. Mounce, R. Jock, C. Bureau-Oxton, P. Harvey-Collard, P. Sharma, J. Anderson, D. Campbell, et al., Single-shot readout performance of two heterojunction-bipolar-transistor amplification circuits at millikelvin temperatures, Sci. Rep. 9, 16976 (2019).

[24] M. G. House, I. Bartlett, P. Pakkiam, M. Koch, E. Peretz, J. vanderHeijden, T. Kobayashi, S. Rogge, and M. Y. Simmons, High-Sensitivity Charge Detection with a SingleLead Quantum Dot for Scalable Quantum Computation, Phys. Rev. Appl. 6, 044016 (2016).

[25] E. Chanrion, D. J. Niegemann, B. Bertrand, C. Spence, B. Jadot, J. Li, P.-A. Mortemousque, L. Hutin, R. Maurand, X. Jehl, M. Sanquer, S. De Franceschi, C. B äuerle, F. Balestro, 
Y.-M. Niquet, M. Vinet, T. Meunier, and M. Urdampilleta, Charge detection in an array of CMOS quantum dots, arXiv:2004.01009.

[26] F. Ansaloni, A. Chatterjee, H. Bohuslavskyi, B. Bertrand, L. Hutin, M. Vinet, and F. Kuemmeth, Single-electron control in a foundry-fabricated two-dimensional qubit array, arXiv:2004.00894.

[27] F. Persson, C. M. Wilson, M. Sandberg, G. Johansson, and P. Delsing, Excess dissipation in a single-electron box: The sisyphus resistance, Nano Lett. 10, 953 (2010).

[28] M. F. Gonzalez-Zalba, S. Barraud, A. J. Ferguson, and A. C. Betz, Probing the limits of gate-based charge sensing, Nat. Commun. 6, 6084 (2015).

[29] B. Voisin, V. H. Nguyen, J. Renard, X. Jehl, S. Barraud, F. Triozon, M. Vinet, I. Duchemin, Y. M. Niquet, S. De Franceschi, and M. Sanquer, Few-electron edge-state quantum dots in a silicon nanowire field-effect transistor, Nano Lett. 14, 2094 (2014).

[30] I. Ahmed, J. A. Haigh, S. Schaal, S. Barraud, Y. Zhu, C. M. Lee, M. Amado, J. W. A. Robinson, A. Rossi, J. J. L. Morton, and M. F. Gonzalez-Zalba, Radio-Frequency Capacitive Gate-Based Sensing, Phys. Rev. Appl. 10, 014018 (2018).

[31] T. Lundberg, J. Li, L. Hutin, B. Bertrand, D. J. Ibberson, C.-M. Lee, D. J. Niegemann, M. Urdampilleta, N. Stelmashenko, T. Meunier, J. W. A. Robinson, L. Ibberson, M. Vinet, Y. M. Niquet, and M. F. Gonzalez-Zalba, A spin quintet in a silicon double quantum dot: spin blockade and relaxation, arXiv:1910.10118.

[32] D. J. Ibberson, L. Bourdet, J. C. Abadillo-Uriel, I. Ahmed, S. Barraud, M. J. Calderón, Y. M. Niquet, and M. F. Gonzalez-Zalba, Electric-field tuning of the valley splitting in silicon corner dots, Appl. Phys. Lett. 113, 053104 (2018).

[33] S. Schaal, I. Ahmed, J. A. Haigh, L. Hutin, B. Bertrand, S. Barraud, M. Vinet, C.-M. Lee, N. Stelmashenko, J. W. A. Robinson, J. Y. Qiu, S. Hacohen-Gourgy, I. Siddiqi, M. F. Gonzalez-Zalba, and J. J. L. Morton, Fast GateBased Readout of Silicon Quantum Dots Using Josephson Parametric Amplification, Phys. Rev. Lett. 124, 67701 (2020).

[34] B. A. Glavin and K. W. Kim, Spin-lattice relaxation in Si quantum dots, Phys. Rev. B 68, 045308 (2003).

[35] C. H. Yang, A. Rossi, R. Ruskov, N. S. Lai, F. A. Mohiyaddin, S. Lee, C. Tahan, G. Klimeck, A. Morello, and A. S. Dzurak, Spin-valley lifetimes in a silicon quantum dot with tunable valley splitting, Nat. Commun. 4, 2069 (2013).

[36] C. Tahan and R. Joynt, Relaxation of excited spin, orbital, and valley qubit states in ideal silicon quantum dots, Phys. Rev. B 89, 075302 (2014).

[37] L. Bourdet and Y. M. Niquet, All-electrical manipulation of silicon spin qubits with tunable spin-valley mixing, Phys. Rev. B 97, 155433 (2018).

[38] A. Corna, L. Bourdet, R. Maurand, A. Crippa, D. KotekarPatil, H. Bohuslavskyi, R. Laviéville, L. Hutin, S. Barraud, X. Jehl, M. Vinet, S. De Franceschi, Y. M. Niquet, and M. Sanquer, Electrically driven electron spin resonance mediated by spin-valley-orbit coupling in a silicon quantum dot, npj Quantum Inf. 4, 6 (2018).
[39] C. Tahan and R. Joynt, Relaxation of excited spin, orbital, and valley qubit states in ideal silicon quantum dots, Phys. Rev. B 89, 075302 (2014).

[40] P. Scarlino, E. Kawakami, P. Stano, M. Shafiei, C. Reichl, W. Wegscheider, and L. M. K. Vandersypen, SpinRelaxation Anisotropy in a GaAs Quantum Dot, Phys. Rev. Lett. 113, 256802 (2014).

[41] K. MacLean, S. Amasha, I. P. Radu, D. M. Zumbühl, M. A. Kastner, M. P. Hanson, and A. C. Gossard, EnergyDependent Tunneling in a Quantum Dot, Phys. Rev. Lett. 98, 036802 (2007).

[42] S. Amasha, K. MacLean, I. P. Radu, D. M. Zumbühl, M. A. Kastner, M. P. Hanson, and A. C. Gossard, Spin-dependent tunneling of single electrons into an empty quantum dot, Phys. Rev. B 78, 041306(R) (2008).

[43] M. Friesen, C. Tahan, R. Joynt, and M. A. Eriksson, Spin Readout and Initialization in a Semiconductor Quantum Dot, Phys. Rev. Lett. 92, 037901 (2004).

[44] C. B. Simmons, J. R. Prance, B. J. Van Bael, T. S. Koh, Z. Shi, D. E. Savage, M. G. Lagally, R. Joynt, M. Friesen, S. N. Coppersmith, and M. A. Eriksson, Tunable Spin Loading and T1 of a Silicon Spin Qubit Measured by Single-Shot Readout, Phys. Rev. Lett. 106, 156804 (2011).

[45] A. Hollmann, T. Struck, V. Langrock, A. Schmidbauer, F. Schauer, T. Leonhardt, K. Sawano, H. Riemann, N. V. Abrosimov, D. Bougeard, and L. R. Schreiber, Large, Tunable Valley Splitting and Single-Spin Relaxation Mechanisms in a $\mathrm{Si} / \mathrm{Si}_{x} \mathrm{Ge}_{1-x}$ Quantum Dot, Phys. Rev. Appl. 13, 034068 (2020).

[46] P. Huang and X. Hu, Spin relaxation in a Si quantum dot due to spin-valley mixing, Phys. Rev. B 90, 235315 (2014).

[47] L. Petit, J. M. Boter, H. G. J. Eenink, G. Droulers, M. L. V. Tagliaferri, R. Li, D. P. Franke, K. J. Singh, J. S. Clarke, R. N. Schouten, V. V. Dobrovitski, L. M. K Vandersypen, and M. Veldhorst, Spin Lifetime and Charge Noise in Hot Silicon Quantum Dot Qubits, Phys. Rev. Lett. 121, 076801 (2018).

[48] X. Zhang, R.-z. Hu, H.-o. Li, F.-m. Jing, Y. Zhou, R.-L. Ma, M. Ni, G. Luo, G. Cao, G.-L. Wang, X. Hu, H.-W. Jiang, G.-C. Guo, and G.-P. Guo, Giant Anisotropy of Spin Relaxation and Spin-Valley Mixing in a Silicon Quantum Dot, Phys. Rev. Lett. 124, 257701 (2020).

[49] F. Borjans, D. M. Zajac, T. M. Hazard, and J. R. Petta, Single-Spin Relaxation in a Synthetic Spin-Orbit Field, Phys. Rev. Appl. 11, 044063 (2019).

[50] M. O. Nestoklon, L. E. Golub, and E. L. Ivchenko, Spin and valley-orbit splittings in SiGe/Si heterostructures, Phys. Rev. B 73, 235334 (2006).

[51] M. Veldhorst, R. Ruskov, C. H. Yang, J. C. C. Hwang, F. E. Hudson, M. E. Flatté, C. Tahan, K. M. Itoh, A. Morello, and A. S. Dzurak, Spin-orbit coupling and operation of multi-valley spin qubits, Phys. Rev. B 92, 201401(R) (2015).

[52] D. Keith, M. G. House, M. B. Donnelly, T. F. Watson, B. Weber, and M. Y. Simmons, Single-Shot Spin Readout in Semiconductors Near the Shot-Noise Sensitivity Limit, Phys. Rev. X 9, 41003 (2019).

[53] D. J. Ibberson, T. Lundberg, J. A. Haigh, L. Hutin, B. Bertrand, S. Barraud, C.-M. Lee, N. A. Stelmashenko, 
J. W. A. Robinson, M. Vinet, M. F. Gonzalez-Zalba, and L. A. Ibberson, Large dispersive interaction between a CMOS double quantum dot and microwave photons, arXiv:2004.00334.

[54] L. Hutin, et al., in 2019 IEEE International Electron Devices Meeting (IEDM) (2019), p. 37.7.1.

[55] Z. Cai, Resource estimation for quantum variational simulations of the Hubbard model: The advantage of multi-core NISQ processing, arXiv:1910.02719.

[56] C. Cade, L. Mineh, A. Montanaro, and S. Stanisic, Strategies for solving the Fermi-Hubbard model on near-term quantum computers, arXiv:1912.06007.

[57] T. Fujita, T. A. Baart, C. Reichl, W. Wegscheider, and L. M. K. Vandersypen, Coherent shuttle of electron-spin states, npj Quantum Inf. 3, 22 (2017).

[58] A. J. Sigillito, M. J. Gullans, L. F. Edge, M. Borselli, and J. R. Petta, Coherent transfer of quantum information in a silicon double quantum dot using resonant SWAP gates, npj Quantum Inf. 5, 110 (2019).

[59] X. Yuan, S. Endo, Q. Zhao, Y. Li, and S. C. Benjamin, Theory of variational quantum simulation, Quantum 3, 191 (2019).

[60] http://mos-quito.eu.
[61] M. Xiao, M. G. House, and H. W. Jiang, Measurement of the Spin Relaxation Time of Single Electrons in a Silicon Metal-Oxide-Semiconductor-Based Quantum Dot, Phys. Rev. Lett. 104, 096801 (2010).

[62] C. A. Ulrich, Semiconductor nanostructures, Lect. Notes Phys. 706, 271 (2006).

[63] W. G. Van der Wiel, S. De Franceschi, J. M. Elzerman, T. Fujisawa, S. Tarucha, and L. P. Kouwenhoven, Electron transport through double quantum dots, Rev. Mod. Phys. 75, 1 (2002).

[64] I. Ahmed, A. Chatterjee, S. Barraud, J. J. L. Morton, J. A. Haigh, and M. F. Gonzalez-Zalba, Primary thermometry of a single reservoir using cyclic electron tunneling to a quantum dot, Commun. Phys. 1, 66 (2018).

[65] B. Venitucci, J. Li, L. Bourdet, and Y. Niquet, in 2019 Int. Conf. on Simulation of Semiconductor Processes and Devices, Udine, Italy (2019), p. 1.

[66] X. Zhang, R. Z. Hu, H. O. Li, F. M. Jing, and Y. Zhou, Giant anisotropy of spin relaxation and spin-valley mixing in a silicon quantum dot, arXiv:1912.08365.

[67] R. Hanson, L. P. Kouwenhoven, J. R. Petta, S. Tarucha, and L. M. K. Vandersypen, Spins in few-electron quantum dots, Rev. Mod. Phys. 79, 1217 (2007). 